\title{
Spectra and ergodic properties of multiplication and convolution operators on the space $\mathcal{S}(\mathbb{R})$
}

\author{
Angela A. Albanese $^{1}\left(\right.$ (i) $\cdot$ Claudio Mele $^{1}$ \\ Received: 24 March 2021 / Accepted: 12 July 2021 / Published online: 30 July 2021 \\ (C) The Author(s) 2021
}

\begin{abstract}
In this paper we investigate the spectra and the ergodic properties of the multiplication operators and the convolution operators acting on the Schwartz space $\mathcal{S}(\mathbb{R})$ of rapidly decreasing functions, i.e., operators of the form $M_{h}: \mathcal{S}(\mathbb{R}) \rightarrow \mathcal{S}(\mathbb{R}), f \mapsto h f$, and $C_{T}: \mathcal{S}(\mathbb{R}) \rightarrow \mathcal{S}(\mathbb{R}), f \mapsto T \star f$. Precisely, we determine their spectra and characterize when those operators are power bounded and mean ergodic.
\end{abstract}

Keywords Rapidly decreasing functions · Multiplication operator · Convolution operator $\cdot$ Spectra $\cdot$ Power bounded operator $\cdot$ Mean ergodic operator

Mathematics Subject Classification Primary 47B38 - 46E10 - 46F05; Secondary $47 \mathrm{~A} 10 \cdot 47 \mathrm{~A} 35$

\section{Introduction}

Convolution operators, as well as multiplication operators, have been intensively studied in spaces of functions or distributions, from different point of views. For instance, the problem to characterize when the multiplication operator acting on smooth functions has closed range has attracted the attention of several authors (see [8] and the references therein) and is still open. The problem is equivalent to the well-known division problem for distributions posed by L. Schwartz [23, Chap. 5, Section 5]. However, it seems that power boundedness and (uniform) mean ergodicity of multiplication and convolution operators on the Schwartz space $\mathcal{S}\left(\mathbb{R}^{N}\right)$ of rapidly decreasing functions had not been investigated.

Angela A. Albanese

angela.albanese@unisalento.it

Claudio Mele

claudio.mele1@unisalento.it

1 Dipartimento di Matematica e Fisica “E. De Giorgi”, Università del Salento- C.P.193, 73100 Lecce, Italy 
The Schwartz space $\mathcal{S}\left(\mathbb{R}^{N}\right)$ of rapidly decreasing functions is the most important space of classical analysis besides the space of smooth functions and the space of real analytic functions. The multipliers of $\mathcal{S}\left(\mathbb{R}^{N}\right)$ are the functions $h \in C^{\infty}\left(\mathbb{R}^{N}\right)$ such that the multiplication operator $M_{h}: \mathcal{S}\left(\mathbb{R}^{N}\right) \mapsto \mathcal{S}\left(\mathbb{R}^{N}\right), f \mapsto h f$, is well defined and continuous. The space of all multipliers is denoted by $\mathcal{O}_{M}\left(\mathbb{R}^{N}\right)$. The convolutors of $\mathcal{S}\left(\mathbb{R}^{N}\right)$ are the distributions $T \in \mathcal{S}^{\prime}\left(\mathbb{R}^{N}\right)$ such that the convolution operator $C_{T}: \mathcal{S}\left(\mathbb{R}^{N}\right) \rightarrow \mathcal{S}\left(\mathbb{R}^{N}\right), f \mapsto T \star f$, is well defined and continuous. The space of all convolutors is denoted by $\mathcal{O}_{C}^{\prime}\left(\mathbb{R}^{N}\right)$. In [8] the authors characterized the multipliers $h \in \mathcal{O}_{M}(\mathbb{R})$ such that $M_{h}: \mathcal{S}(\mathbb{R}) \rightarrow \mathcal{S}(\mathbb{R})$ has closed range. We also mention that in the last years the study of the properties, like closed range and dynamical behaviour, of the composition operators acting on the Schwartz space $\mathcal{S}(\mathbb{R})$ has been considered by several authors (see [10-13] for examples and the references therein).

In this paper we study the spectra and the ergodic properties of the multiplication and the convolution operators defined in the Schwartz space $\mathcal{S}(\mathbb{R})$ of one variable rapidly decreasing functions. We determine their spectra and characterize when those operators are power bounded and (uniformly) mean ergodic. In particular, we show that the spectra of the multiplication operator $M_{h}$ (the convolution operator $C_{T}$, resp.) acting either on $\mathcal{S}(\mathbb{R})$ or on $\mathcal{O}_{M}(\mathbb{R})\left(\right.$ on $\mathcal{O}_{C}(\mathbb{R})$, the strong dual of $\mathcal{O}_{C}^{\prime}(\mathbb{R})$, resp.) coincide, Theorems 3.4 and 3.6. We prove that the multiplication operator $M_{h}$ is power bounded (uniformly mean ergodic, resp.) when it acts on $\mathcal{S}(\mathbb{R})$ if and only if it is power bounded (uniformly mean ergodic, resp.) when it acts on $\mathcal{O}_{M}(\mathbb{R})$, Theorems 4.3 and 4.4. These conditions are expressed in terms of the multiplier $h$. Similar characterizations are also given in the case of convolution operators. The properties of the Fourier transform allow to reduce the proofs to the multiplication operator case. Precisely, we show that the convolution operator $C_{T}$ is power bounded (uniformly mean ergodic, resp.) when it acts on $\mathcal{S}(\mathbb{R})$ if and only if it is power bounded (uniformly mean ergodic, resp.) when it acts on $\mathcal{O}_{C}(\mathbb{R})$, Propositions 4.7 and 4.8. These conditions are expressed in terms of the convolutor $T$.

We also present characterizations of the power boundedness and the (uniform) mean ergodicity of the multiplication operators and the convolution operators when those act on the space $C^{\infty}(\mathbb{R})$, Propositions 4.1 and 4.10.

The paper ends with an "Appendix", where we collect some general results on the spectrum of operators acting on Fréchet spaces.

We remark that the description of the spectra and the characterizations of the power boundedness and the mean ergodicity are valid for the several variables case with the same proofs. We have considered only the one dimensional case for the sake of simplicity in computations of derivatives to describe the spectra.

\section{Preliminaries}

In this section, we first recall some general notation and results on operators in locally convex spaces.

Let $E$ be a locally convex Hausdorff space (briefly, lcHs) and let $\mathcal{L}(E)$ denote the space of all continuous linear operators from $E$ into itself. Given $T \in \mathcal{L}(E)$, the 
resolvent set of $T$ is defined by

$$
\rho(T):=\left\{\lambda \in \mathbb{C}: \lambda I-T: E \rightarrow E \text { is bijective and }(\lambda I-T)^{-1} \in \mathcal{L}(E)\right\}
$$

and the spectrum of $T$ is defined by $\sigma(T):=\mathbb{C} \backslash \rho(T)$. For $\lambda \in \rho(T)$ we define $R(\lambda, T):=(\lambda I-T)^{-1}$ which is called the resolvent operator of $T$ at $\lambda$. The point spectrum is defined by

$$
\sigma_{p}(T):=\{\lambda \in \mathbb{C}: \lambda I-T \text { is not injective }\}
$$

Whenever $\lambda, \mu \in \rho(T)$ we have the resolvent identity $R(\lambda, T)-R(\mu, T)=(\mu-$ $\lambda) R(\lambda, T) R(\mu, T)$. Unlike for Banach spaces, it may happens that $\rho(T)=\emptyset$ or that $\rho(T)$ is not open in $\mathbb{C}$ (see, f.i., [7]). This is the reason for which many authors consider the subset $\rho^{*}(T)$ of $\rho(T)$ consisting of all $\lambda \in \mathbb{C}$ for which there exists $\delta>0$ such that $B(\lambda, \delta):=\{\mu \in \mathbb{C}:|\mu-\lambda|<\delta\} \subseteq \rho(T)$ and the set $\{R(\mu, T): \mu \in B(\lambda, \delta)\}$ is equicontinuous in $\mathcal{L}(E)$. If $E$ is a Fréchet space, then it suffices that this set is bounded in $\mathcal{L}_{S}(E)$, where $\mathcal{L}_{S}(E)$ denotes $\mathcal{L}(E)$ endowed with the strong operator topology, i.e., the topology of uniform convergence on the finite subsets of $E$. The advantange of $\rho^{*}(T)$, whenever it is not empty, is that it is open and the resolvent map $R: \lambda \mapsto R(\lambda, T)$ is holomorphic from $\rho^{*}(T)$ into $\mathcal{L}_{b}(E)$ (see, f.i., [5, Propositione 3.4]), where $\mathcal{L}_{b}(E)$ denotes $\mathcal{L}(E)$ endowed with the topology of uniform convergence on the bounded subsets of $E$. Define $\sigma^{*}(T):=\mathbb{C} \backslash \rho^{*}(T)$, which is a closed set containing $\sigma(T)$. In [5, Remark 3.5(vi)] an example of an operator $T \in \mathcal{L}(E)$, with $E$ a Fréchet space, is presented such that $\overline{\sigma(T)} \subsetneq \sigma^{*}(T)$.

For further basic properties of the resolvent set and the resolvent map we refer to $[24,25]$ for operators on locally convex spaces.

An operator $T \in \mathcal{L}(E)$, with $E$ a lcHs, is called power bounded if $\left\{T^{n}\right\}_{n \in \mathbb{N}}$ is an equicontinuous subset of $\mathcal{L}(E)$.

The Cesàro means of an operators $T \in \mathcal{L}(E)$, with $E$ a lcHs, are defined by

$$
T_{[n]}:=\frac{1}{n} \sum_{m=1}^{n} T^{m}, \quad n \in \mathbb{N} .
$$

The operator $T$ is called mean ergodic (resp. uniformly mean ergodic) if $\left\{T_{[n]}\right\}_{n \in \mathbb{N}}$ is a convergent sequence in $\mathcal{L}_{s}(E)$ (resp. in $\mathcal{L}_{b}(E)$ ). The Cesàro means of $T$ satisfy the following identities

$$
\frac{T^{n}}{n}=T_{[n]}-\frac{n-1}{n} T_{[n-1]}, \quad n \geq 2 .
$$

So, it is clear that $\frac{T^{n}}{n} \rightarrow 0$ in $\mathcal{L}_{s}(E)$ as $n \rightarrow \infty$, whenever $T$ is mean ergodic. Furthemore, if $E$ is a barrelled lcHs space and $T \in \mathcal{L}(E)$ is mean ergodic, the operator $P:=\lim _{n \rightarrow \infty} T_{[n]}$ in $\mathcal{L}_{S}(E)$ is a projection on $E$ satisfying $\operatorname{Im} P=\operatorname{ker}(I-T)$ and ker $P=\overline{\operatorname{Im}(I-T)}$ with

$$
X=\overline{\operatorname{Im}(I-T)} \oplus \operatorname{ker}(I-T) .
$$


If $E$ is a Montel lcHs, i.e., a barrelled lcHs such that every bounded set is relatively compact, then the operator $T$ is uniformly mean ergodic whenever it is mean ergodic. Furthemore, in reflexive Fréchet spaces (in Montel Fréchet spaces, resp.) every power bounded operator is necessarily mean ergodic (uniformly mean ergodic, resp.) [1, Corollary 2.7, Proposition 2.9]. The converse is not true in general, see, f.i., [15, §6].

For further results on mean ergodic operators we refer to [19,26]. For recent results on mean ergodic operators in lcHs' we refer to [1,2,4-6,22], for example, and the references therein.

We now recall the necessary definitions and some basic properties of the space $\mathcal{S}(\mathbb{R})$ and the spaces $\mathcal{O}_{M}(\mathbb{R})$ and $\mathcal{O}_{C}(\mathbb{R})$.

The space $\mathcal{S}(\mathbb{R})$ of rapidly decreasing functions on $\mathbb{R}$ is defined by

$$
\begin{aligned}
\mathcal{S}(\mathbb{R})=\left\{f \in C^{\infty}(\mathbb{R}):\|f\|_{n}:=\right. & \sup _{x \in \mathbb{R}} \sup _{i=0, \ldots, n}\left(1+x^{2}\right)^{n}\left|f^{(i)}(x)\right| \\
& \left.<+\infty \text { for every } n \in \mathbb{N}_{0}\right\} .
\end{aligned}
$$

The space $\mathcal{S}(\mathbb{R})$ is a nuclear Fréchet space and hence, it is Montel and reflexive. Accordingly, its strong dual $\mathcal{S}^{\prime}(\mathbb{R})$ is a nuclear lcHs. In particular, $\mathcal{S}^{\prime}(\mathbb{R})$ is a barrelled and bornological lcHs.

The space $\mathcal{O}_{M}(\mathbb{R})$ of slowly increasing functions on $\mathbb{R}$ is given by

$$
\mathcal{O}_{M}(\mathbb{R})=\cap_{m=1}^{\infty} \cup_{n=1}^{\infty}\left\{f \in C^{\infty}(\mathbb{R}):|f|_{m, n}:=\sup _{x \in \mathbb{R}} \sup _{0 \leq i \leq m}\left(1+x^{2}\right)^{-n}\left|f^{(i)}(x)\right|<\infty\right\},
$$

where $\mathcal{O}_{n}^{m}(\mathbb{R}):=\left\{f \in C^{\infty}(\mathbb{R}):|f|_{m, n}:=\sup _{x \in \mathbb{R}} \sup _{0 \leq i \leq m}\left(1+x^{2}\right)^{-n}\left|f^{(i)}(x)\right|<\right.$ $\infty$, endowed with the norm $|\cdot|_{m, n}$, is a Banach space for any $m, n \in \mathbb{N}$. The space $\mathcal{O}_{M}(\mathbb{R})$, endowed with its natural lc-topology, i.e., $\mathcal{O}_{M}(\mathbb{R})=\operatorname{proj}_{m}^{m}$ ind ${ }_{n}^{\rightarrow} \mathcal{O}_{n}^{m}(\mathbb{R})$, is a projective limit of complete (LB)-spaces. In particular, $\mathcal{O}_{M}(\mathbb{R})$ is a bornological nuclear lcHs (hence, Montel and reflexive), see [14]. Its strong dual $\mathcal{O}_{M}^{\prime}(\mathbb{R})$ is also a bornological nuclear lcHs. Furthemore, a fundamental system of continuous norms on $\mathcal{O}_{M}(\mathbb{R})$ is given by

$$
p_{m, v}(f)=\sup _{x \in \mathbb{R}} \sup _{0 \leq i \leq m}|v(x)|\left|f^{(i)}(x)\right|, \quad f \in \mathcal{O}_{M}(\mathbb{R}),
$$

where $v \in \mathcal{S}(\mathbb{R})$ and $m \in \mathbb{N}$ (see, f.i., [9]). Since $\left(\mathcal{O}_{M}(\mathbb{R}), \cdot\right)$ is an algebra, we have then for every $m \in \mathbb{N}$ and $v \in \mathcal{S}(\mathbb{R})$ that there exist $v_{1}, v_{2} \in \mathcal{S}(\mathbb{R})$ and $m^{\prime} \in \mathbb{N}$ with $m^{\prime} \geq m$ such that

$$
p_{m, v}(f g) \leq p_{m^{\prime}, v_{1}}(f) p_{m^{\prime}, v_{2}}(g), \quad f, g \in \mathcal{O}_{M}(\mathbb{R})
$$

The space $\mathcal{O}_{C}(\mathbb{R})$ of very slowly increasing functions on $\mathbb{R}$ is given by

$$
\mathcal{O}_{C}(\mathbb{R})=\cup_{n=1}^{\infty} \cap_{m=1}^{\infty}\left\{f \in C^{\infty}(\mathbb{R}):|f|_{m, n}:=\sup _{x \in \mathbb{R}} \sup _{0 \leq i \leq m}\left(1+x^{2}\right)^{-n}\left|f^{(i)}(x)\right|<\infty\right\} .
$$

The space $\mathcal{O}_{C}(\mathbb{R})$, endowed with its natural lc-topology, i.e., $\mathcal{O}_{C}(\mathbb{R})=$ ind $\stackrel{n}{\rightarrow}$ $\operatorname{proj} \underset{\leftarrow}{m} \mathcal{O}_{n}^{m}(\mathbb{R})$, is a complete $(\mathrm{LF})$-space. In particular, $\mathcal{O}_{C}(\mathbb{R})$ is a bornological nuclear 
lcHs (hence, Montel and reflexive), see [14]. Its strong dual $\mathcal{O}_{C}^{\prime}(\mathbb{R})$ is the space of all convolutors of $\mathcal{S}(\mathbb{R})$. In particular, $\mathcal{O}_{C}^{\prime}(\mathbb{R})$ is also a bornological nuclear lcHs.

The space $\mathcal{O}_{M}(\mathbb{R})$ is the space of multipliers of $\mathcal{S}(\mathbb{R})$ and its strong dual $\mathcal{S}^{\prime}(\mathbb{R})$. So, for any fixed $h \in \mathcal{O}_{M}(\mathbb{R})$, the multiplication operator $M_{h}: \mathcal{S}(\mathbb{R}) \rightarrow \mathcal{S}(\mathbb{R}), f \mapsto h f$, is continuous and hence, its transpose $\mathcal{M}_{h}:=M_{h}^{\prime}: \mathcal{S}^{\prime}(\mathbb{R}) \rightarrow \mathcal{S}^{\prime}(\mathbb{R}), S \mapsto h S$, is also continuous. Furthemore, for any $h \in \mathcal{O}_{M}(\mathbb{R})$, the multiplication operator $M_{h}\left(\mathcal{M}_{h}\right.$, resp.) acts continuosly from $\mathcal{O}_{M}(\mathbb{R})$ into itself (from $\mathcal{O}_{M}^{\prime}(\mathbb{R})$ into itself, resp.).

The space $\mathcal{O}_{C}^{\prime}(\mathbb{R})$ is the space of convolutors of $\mathcal{S}(\mathbb{R})$ and its strong dual $\mathcal{S}^{\prime}(\mathbb{R})$. Precisely, if $T \in \mathcal{O}_{C}^{\prime}(\mathbb{R})$ and $f \in \mathcal{S}(\mathbb{R})$, then the convolution $T \star f$ defined by

$$
(T \star f)(x):=\left\langle T_{y}, \widetilde{\tau_{x}} f\right\rangle, \quad x \in \mathbb{R},
$$

is a function of $\mathcal{S}(\mathbb{R})$, where $\left(\tau_{x} f\right)(y):=f(x+y)$ for $x, y \in \mathbb{R}$ and $\check{f}(y):=f(-y)$ for $y \in \mathbb{R}$. While, if $T \in \mathcal{O}_{C}^{\prime}(\mathbb{R})$ and $S \in \mathcal{S}^{\prime}(\mathbb{R})$, then the convolution $T \star S$ defined by

$$
(T \star S)(f)=\langle S, \check{T} \star f\rangle, \quad f \in \mathcal{S}(\mathbb{R}),
$$

belongs to $\mathcal{S}^{\prime}(\mathbb{R})$, where $\check{T}$ denotes the distribution defined by $\varphi \mapsto\langle\check{T}, \varphi\rangle:=\langle T, \check{\varphi}\rangle$. We point out that in case $S$ belongs to $\mathcal{O}_{C}^{\prime}(\mathbb{R})$, the convolution $T \star S \in \mathcal{O}_{C}^{\prime}(\mathbb{R})$ too.

For any fixed $T \in \mathcal{O}_{C}^{\prime}(\mathbb{R})$, the convolution operator $C_{T}: \mathcal{S}(\mathbb{R}) \rightarrow \mathcal{S}(\mathbb{R})$ (the convolution operator $C_{T}: \mathcal{O}_{C}(\mathbb{R}) \rightarrow \mathcal{O}_{C}(\mathbb{R})$, resp.), $f \mapsto T \star f$, is a continuous linear operator from $\mathcal{S}(\mathbb{R})$ into itself (from $\mathcal{O}_{C}(\mathbb{R})$ into itself, resp.) and hence, its transpose $\mathcal{C}_{T}:=C_{T}^{\prime}: \mathcal{S}^{\prime}(\mathbb{R}) \rightarrow \mathcal{S}^{\prime}(\mathbb{R})\left(\mathcal{C}_{T}:=C_{T}^{\prime}: \mathcal{O}_{C}^{\prime}(\mathbb{R}) \rightarrow \mathcal{O}_{C}^{\prime}(\mathbb{R})\right.$, resp. $), S \mapsto T \star S$, is also a continuous linear operator from $\mathcal{S}^{\prime}(\mathbb{R})$ into itself (from $\mathcal{O}_{C}^{\prime}(\mathbb{R})$ into itself, resp.).

Through the paper, we consider the following notation for the Fourier transform of a function $f \in L^{1}(\mathbb{R})$ :

$$
\hat{f}(\xi):=\mathcal{F}(f)(\xi)=\int_{\mathbb{R}} e^{-i x \xi} f(x) d x, \quad \xi \in \mathbb{R} .
$$

The Fourier transform $\mathcal{F}: \mathcal{S}(\mathbb{R}) \rightarrow \mathcal{S}(\mathbb{R})$ is a topological isomorphism from $\mathcal{S}(\mathbb{R})$ onto itself, that can be extended in the usual way to $\mathcal{S}^{\prime}(\mathbb{R})$, i.e. $\mathcal{F}(T)(f):=\langle T, \hat{f}\rangle$ for every $f \in \mathcal{S}(\mathbb{R})$ and $T \in \mathcal{S}^{\prime}(\mathbb{R})$. Furthemore, the Fourier transfom $\mathcal{F}$ is a topological isomorphism from the space $\mathcal{O}_{C}^{\prime}(\mathbb{R})$ onto the space $\mathcal{O}_{M}(\mathbb{R})$.

For further properties on the spaces $\mathcal{O}_{M}(\mathbb{R})$ and $\mathcal{O}_{C}(\mathbb{R})$ and the Fourier transform we refer, for instance, to $[9,14,16,23]$ (see also $[20,21]$ and the references therein).

\section{Spectra of multiplication and convolution operators on $\mathcal{S}(\mathbb{R})$}

The aim of this section is to study the spectra of multiplication operators $M_{h}$, for $h \in \mathcal{O}_{M}(\mathbb{R})$, and convolution operators $C_{T}$, for $T \in \mathcal{O}_{C}^{\prime}(\mathbb{R})$, when acting on the space $\mathcal{S}(\mathbb{R})$.

To this target, we begin by stating and proving some auxiliary results. 
Lemma 3.1 Let $h \in \mathcal{O}_{M}(\mathbb{R})$ such that $0 \notin \overline{\operatorname{Im} h}$. Then $\frac{1}{h} \in \mathcal{O}_{M}(\mathbb{R})$.

Proof According to the Faà Bruno formula, we have for every $x \in \mathbb{R}$ and $n \in \mathbb{N}_{0}$ that

$$
\left(\frac{1}{h}\right)^{(n)}(x)=\sum \frac{n !}{k_{1} ! k_{2} ! \ldots k_{n} !} \frac{(-1)^{k} k !}{(h(x))^{k+1}}\left(\frac{h^{\prime}(x)}{1 !}\right)^{k_{1}}\left(\frac{h^{\prime \prime}(x)}{2 !}\right)^{k_{2}} \ldots\left(\frac{h^{(n)}(x)}{n !}\right)^{k_{n}}
$$

where the sum is extended over all $\left(k_{1}, k_{2}, \ldots, k_{n}\right) \in \mathbb{N}_{0}^{n}$ such that $k_{1}+2 k_{2}+\ldots+$ $n k_{n}=n$ and $k_{1}+k_{2}+\ldots+k_{n}=k$ (hence, $k_{1}+k_{2}+\ldots+k_{n} \leq n$ ). Since $0 \notin \overline{\operatorname{Im} h}$, it clearly follows that $\frac{1}{h} \in C^{\infty}(\mathbb{R})$. So, it remains to show that $\frac{1}{h} \in \mathcal{O}_{M}(\mathbb{R})$. To this end, let choose $d>0$ such that $d<\min \{1, d(0, \overline{\operatorname{Im} h})\}$ and fix $l \in \mathbb{N}$. Since $h \in \mathcal{O}_{M}(\mathbb{R})$ there exist $C \geq 1$ and $j \in \mathbb{N}$ such that

$$
\left|h^{(i)}(x)\right| \leq C\left(1+x^{2}\right)^{j}, \quad x \in \mathbb{R}, i=0,1, \ldots, l .
$$

So, we have for every $x \in \mathbb{R}$ and $n=0,1, \ldots, l$ that

$$
\begin{aligned}
\left|\left(\frac{1}{h}\right)^{(n)}(x)\right| & \leq \sum \frac{n !}{k_{1} ! k_{2} ! \ldots k_{n} !} \frac{k !}{|h(x)|^{k+1}} \frac{C^{k}\left(1+x^{2}\right)^{j k}}{(1 !)^{k_{1}}(2 !)^{k_{2}} \ldots(n !)^{k_{n}}} \\
& \leq \frac{C^{n}}{d^{n+1}}\left(1+x^{2}\right)^{j n} \sum \frac{n ! k !}{k_{1} ! k_{2} ! \ldots k_{n} !(1 !)^{k_{1}}(2 !)^{k_{2}} \ldots(n !)^{k_{n}}} \\
& \leq \frac{C^{l} D}{d^{l+1}}\left(1+x^{2}\right)^{j l}
\end{aligned}
$$

where $D:=\max \left\{\sum \frac{n ! k !}{k_{1} ! k_{2} ! \ldots . k_{n} !} \frac{1}{(1 !)^{k_{1}}(2 !)^{k_{2}} \ldots(n !)^{k_{n}}}: n=0, \ldots, l\right\}<+\infty$. Since $l \in \mathbb{N}$ is arbitrary, this implies that $\frac{1}{h} \in \mathcal{O}_{M}(\mathbb{R})$.

Proposition 3.2 Let $h \in \mathcal{O}_{M}(\mathbb{R})$. Then the following properties are equivalent.

(1) $M_{h}: \mathcal{S}(\mathbb{R}) \rightarrow \mathcal{S}(\mathbb{R})$ is surjective.

(2) $M_{h}: \mathcal{O}_{M}(\mathbb{R}) \rightarrow \mathcal{O}_{M}(\mathbb{R})$ is surjective.

(3) $0 \notin \operatorname{Im} h$ and $\frac{1}{h} \in \mathcal{O}_{M}(\mathbb{R})$.

(4) There exist $j \in \mathbb{N}$ and $c>0$ such that $|h(x)| \geq \frac{c}{\left(1+x^{2}\right)^{j}}$ for every $x \in \mathbb{R}$.

Proof $(3) \Rightarrow(1)$ and $(3) \Rightarrow(2)$ are obviuos.

$(1) \Rightarrow(3)$. Since $M_{h}$ is surjective, its range $\operatorname{Im} M_{h}=\mathcal{S}(\mathbb{R})$ is clearly a closed subspace of $\mathcal{S}(\mathbb{R})$. Thus, $M_{h}$ is also injective. So, $M_{h}$ is a topological isomorphism from $\mathcal{S}(\mathbb{R})$ onto itself.

Suppose that $0 \in \operatorname{Im} h$, i.e., that there exists $x_{0} \in \mathbb{R}$ such that $h\left(x_{0}\right)=0$. Then the functions $g \in \mathcal{S}(\mathbb{R})$ that do not vanish at $x_{0}$ are not in the range of the operator $M_{h}$. This is a contradiction.

The fact that $0 \notin \operatorname{Im} h$ clearly implies $\frac{1}{h} \in C^{\infty}(\mathbb{R})$. Since $M_{h}$ is bijective (actually, it suffices the surjectivity), we have also that $\frac{1}{h} g \in \mathcal{S}(\mathbb{R})$ for every $g \in \mathcal{S}(\mathbb{R})$. This means that $\frac{1}{h} \in \mathcal{O}_{M}(\mathbb{R})$. 
$(2) \Rightarrow(3)$. Since $M_{h}$ is surjective and the function $\mathbf{1}(x):=1$ for $x \in \mathbb{R}$ belongs to $\mathcal{O}_{M}(\mathbb{R})$, there exists $f \in \mathcal{O}_{M}(\mathbb{R})$ such that $M_{h} f=\mathbf{1}$, i.e., $f(x) h(x)=1$ for every $x \in \mathbb{R}$. This necessarily implies that $0 \notin \operatorname{Im} h$ and $f=\frac{1}{h}$. Accordingly, $\frac{1}{h} \in \mathcal{O}_{M}(\mathbb{R})$.

$(3) \Rightarrow(4)$. By assumption the function $\frac{1}{h} \in \mathcal{O}_{M}(\mathbb{R})$. Therefore, for $l=0$ there exist $C>0$ and $j \in \mathbb{N}$ such that for every $x \in \mathbb{R}$

$$
\left|\frac{1}{h(x)}\right| \leq C\left(1+x^{2}\right)^{j}
$$

Accordingly, we get for every $x \in \mathbb{R}$ that

$$
|h(x)| \geq \frac{1}{C} \frac{1}{\left(1+x^{2}\right)^{j}}
$$

$(4) \Rightarrow(3)$. Since $|h(x)| \geq \frac{c}{\left(1+x^{2}\right)^{j}}$ for every $x \in \mathbb{R}$ and some $c>0$ and $j \in \mathbb{N}$, necessarily $0 \notin \operatorname{Im} h$. So, it remains to show that $\frac{1}{h} \in \mathcal{O}_{M}(\mathbb{R})$. To this end, we set $f(x):=\frac{1}{h(x)}$ for $x \in \mathbb{R}$. Hence, according to the Faà Bruno formula, we have for every $n \in \mathbb{N}$ and $x \in \mathbb{R}$ that

$$
f^{(n)}(x)=\sum \frac{n !}{k_{1} ! k_{2} ! \ldots k_{n} !} \frac{(-1)^{k} k !}{(h(x))^{k+1}}\left(\frac{h^{\prime}(x)}{1 !}\right)^{k_{1}}\left(\frac{h^{\prime \prime}(x)}{2 !}\right)^{k_{2}} \ldots\left(\frac{h^{(n)}(x)}{n !}\right)^{k_{n}}
$$

where the sum is extended over all $\left(k_{1}, k_{2}, \ldots, k_{n}\right) \in \mathbb{N}_{0}^{n}$ such that $k_{1}+2 k_{2}+\ldots+$ $n k_{n}=n$ and $k_{1}+k_{2}+\ldots+k_{n}=k$ (hence, $k_{1}+k_{2}+\ldots+k_{n} \leq n$ ). But, $h \in \mathcal{O}_{M}(\mathbb{R})$. So, for a fixed $l \in \mathbb{N}$ there exist $C>0$ and $s \in \mathbb{N}$ such that

$$
\left|h^{(i)}(x)\right| \leq C\left(1+x^{2}\right)^{s}, \quad x \in \mathbb{R}, i=0,1, \ldots, l .
$$

Therefore, it follows for every $x \in \mathbb{R}$ and $n=0,1, \ldots, l$ that

$$
\begin{aligned}
\left|f^{(n)}(x)\right| & \leq \sum \frac{n ! k !}{k_{1} ! k_{2} ! \ldots k_{n} !} c^{-(k+1)}\left(1+x^{2}\right)^{j(k+1)} \frac{C^{k}\left(1+x^{2}\right)^{s k}}{(1 !)^{k_{1}}(2 !)^{k_{2}} \ldots(n !)^{k_{n}}} \\
& \leq\left(\frac{C}{c}\right)^{n+1}\left(1+x^{2}\right)^{n(s+j)+j} \sum \frac{n ! k !}{k_{1} ! k_{2} ! \ldots k_{n} !(1 !)^{k_{1}}(2 !)^{k_{2}} \ldots(n !)^{k_{n}}} \\
& \leq\left(\frac{C}{c}\right)^{l+1} D\left(1+x^{2}\right)^{l(s+j)+j}
\end{aligned}
$$

where $D:=\max \left\{\sum \frac{n ! k !}{k_{1} ! k_{2} ! \ldots k_{n} !} \frac{1}{(1 !)^{k_{1}}(2 !)^{k_{2}} \ldots(n !)^{k_{n}}}: n=0,1, \ldots, l\right\}<+\infty$ (here, without loss of generality, we have assumed that $C>1$ and $0<c<1$ ). Since $l \in \mathbb{N}$ is arbitrary, this means that $\frac{1}{h}=f \in \mathcal{O}_{M}(\mathbb{R})$.

Remark 3.3 We observe that the condition $0 \notin \operatorname{Im} h$ does not imply that $M_{h}$ is surjective. Indeed, consider the function $h(x)=e^{-x^{2}}$ for $x \in \mathbb{R}$. Then $\left.\left.0 \notin \operatorname{Im} h=\right] 0,1\right]$. 
But, $M_{h}: \mathcal{S}(\mathbb{R}) \rightarrow \mathcal{S}(\mathbb{R})$ is not surjective. Otherwise, by Lemma 3.2 the function $\frac{1}{h(x)}=e^{x^{2}}$, for $x \in \mathbb{R}$, should belong to $\mathcal{O}_{M}(\mathbb{R})$. This is false.

We are ready to study the spectra of the multiplication operators acting on $\mathcal{S}(\mathbb{R})$.

Theorem 3.4 Let $h \in \mathcal{O}_{M}(\mathbb{R})$. Then the spectra of the multiplication operator $M_{h}$ acting either on $\mathcal{S}(\mathbb{R})$ or on $\mathcal{O}_{M}(\mathbb{R})$ are given by:

$$
\begin{array}{r}
\operatorname{Im} h \subseteq \sigma\left(M_{h}\right) \subseteq \overline{\operatorname{Im} h}, \sigma^{*}\left(M_{h}\right)=\overline{\operatorname{Im} h} \\
\sigma_{p}\left(M_{h}\right)=\left\{\lambda \in \mathbb{C}: h^{-1}(\lambda) \text { has a non empty interior }\right\} \subseteq \operatorname{Im} h
\end{array}
$$

Proof We first consider the case $M_{h}: \mathcal{S}(\mathbb{R}) \rightarrow \mathcal{S}(\mathbb{R})$. To achieve the result, it suffices to show that $(\overline{\operatorname{Im} h})^{c} \subseteq \rho\left(M_{h}\right) \subseteq(\operatorname{Im} h)^{c}$. So, let $\lambda \notin \overline{\operatorname{Im} h}$ and let $0<2 d<\min \{1, d(\lambda, \overline{\operatorname{Im} h})\}$. Then $d(B(\lambda, d), \overline{\operatorname{Im} h}) \geq d$, thereby implying for every $\mu \in B(\lambda, d)$ that $0 \notin \overline{\operatorname{Im}(\mu-h)}$. So, by Lemma 3.1 for every $\mu \in B(\lambda, d)$ the function $\frac{1}{\mu-h} \in \mathcal{O}_{M}(\mathbb{R})$ and hence, $M_{\frac{1}{\mu-h}} \in \mathcal{L}(S(\mathbb{R}))$. Since $\left(\mu I-M_{h}\right) M_{\frac{1}{\mu-h}}=$ $I=M_{\frac{1}{\mu-h}}\left(\mu I-M_{h}\right)$ for $\mu \in B(\lambda, d)$, it follows that $B(\lambda, d) \subseteq \rho\left(M_{h}\right)$. It remains to show that the set $\left\{M_{\frac{1}{\mu-h}}: \mu \in B(\lambda, \mu)\right\}$ is equicontinuous in $\mathcal{L}(\mathcal{S}(\mathbb{R}))$. To do this, we recall that $h \in \mathcal{O}_{M}(\mathbb{R})$ and hence, for a fixed $l \in \mathbb{N}$ there exist $C \geq 1$ and $j \in \mathbb{N}$ such that

$$
\left|h^{(i)}(x)\right| \leq C\left(1+x^{2}\right)^{j}, \quad x \in \mathbb{R}, i=0,1, \ldots, l .
$$

Now, by proceeding as in the proof of Lemma 3.1, we obtain for every $x \in \mathbb{R}$, $n=0,1, \ldots, l$ and $\mu \in B(\lambda, d)$ that

$$
\left|\left(\frac{1}{\mu-h(x)}\right)^{(n)}\right| \leq \frac{C^{l} D}{d^{l+1}}\left(1+x^{2}\right)^{j l},
$$

where $D>0$ is a constant independent of $\mu$ and $h$. Applying (3.3), we obtain for every $\mu \in B(\lambda, d), g \in \mathcal{S}(\mathbb{R})$ and $n=0, \ldots, l, x \in \mathbb{R}$ that

$$
\begin{aligned}
\left(1+x^{2}\right)^{l}\left|\left(M_{\frac{1}{\mu-h}} g(x)\right)^{(n)}\right| & \leq \sum_{i=0}^{n}\left(\begin{array}{l}
n \\
i
\end{array}\right)\left|\left(\frac{1}{\mu-h(x)}\right)^{(n-i)}\right|\left(1+x^{2}\right)^{l}\left|g^{(i)}(x)\right| \\
& \leq \sum_{i=0}^{n}\left(\begin{array}{l}
n \\
i
\end{array}\right) \frac{C^{l} D}{d^{l+1}}\left(1+x^{2}\right)^{(j+1) l}\left|g^{(i)}(x)\right| \leq \frac{(2 C)^{l} D}{d^{l+1}}\|g\|_{(j+1) l},
\end{aligned}
$$

and hence,

$$
\left\|M_{\frac{1}{\mu-h}} g\right\|_{l} \leq \frac{(2 C)^{l} D}{d^{l+1}}\|g\|_{(j+1) l} .
$$

Since $l \in \mathbb{N}$ is arbitrary, from (3.4) it follows that the set $\left\{M_{\frac{1}{\mu-h}}: \mu \in B(\lambda, \mu)\right\}$ is equicontinuous in $\mathcal{L}(\mathcal{S}(\mathbb{R}))$. So, we have established that $(\overline{\operatorname{Im} h})^{c} \subseteq \rho^{*}\left(M_{h}\right) \subseteq$ $\rho\left(M_{h}\right)$. 
Let $\lambda \in \rho\left(M_{h}\right)$. Then the operator $M_{\lambda-h}$ is a topological isomorphism from $\mathcal{S}(\mathbb{R})$ onto itself and hence, it is surjective. By Proposition 3.2 it follows that $0 \notin \operatorname{Im}(\lambda-h)$ and hence $\lambda \notin \operatorname{Im} h$. So, we have established that $\rho\left(M_{h}\right) \subseteq(\operatorname{Im} h)^{c}$.

Now, from $(\overline{\operatorname{Im} h})^{c} \subseteq \rho^{*}\left(M_{h}\right) \subseteq \rho\left(M_{h}\right) \subseteq(\operatorname{Im} h)^{c}$ if follows that $\operatorname{Im} h \subseteq$ $\sigma\left(M_{h}\right) \subseteq \sigma^{*}\left(M_{h}\right) \subseteq \overline{\operatorname{Im} h}$. Since $\sigma^{*}\left(M_{h}\right)$ is closed, this yields that $\sigma^{*}\left(M_{h}\right)=\overline{\operatorname{Im} h}$ and hence, $\rho^{*}\left(M_{h}\right)=(\overline{\operatorname{Im} h})^{c}$.

Finally, suppose that $\left(\lambda I-M_{h}\right) g=0$ for some $\lambda \in \mathbb{C}$ and $g \in \mathcal{S}(\mathbb{R})$ with $g \neq 0$. Since $g \neq 0$, there exists an open subset $U$ of $\mathbb{R}$ such that $g(x) \neq 0$ whenever $x \in U$. So, $h(x)=\lambda$ for every $x \in U$, thereby implying that $h^{-1}(\lambda)$ has a non empty interior. Conversely, if $h^{-1}(\lambda)$ has a non empty interior, then any function $g \in \mathcal{D}(U), g \neq 0$, with $U$ the interior set of $h^{-1}(\lambda)$, clearly satisfies $\left(\lambda I-M_{h}\right) g=0$, i.e., $\lambda \in \sigma_{p}\left(M_{h}\right)$. This completes the proof.

The proof for the case $M_{h}: \mathcal{O}_{M}(\mathbb{R}) \rightarrow \mathcal{O}_{M}(\mathbb{R})$ follows by arguing as above with some obvious changes. We only prove that $(\overline{\operatorname{Im} h})^{c} \subseteq \rho^{*}\left(M_{h}\right)$, i.e., that $\sigma^{*}\left(M_{h}\right) \subseteq$ $\overline{\operatorname{Im} h}$. So, let $\lambda \notin \overline{\operatorname{Im} h}$ and let $0<2 d<\min \{1, d(\lambda, \overline{\operatorname{Im} h})\}$. Then for a fixed $l \in \mathbb{N}$, the same arguments above show that inequality (3.3) is valid for every $x \in \mathbb{R}$, $n=0, \ldots, l$ and $\mu \in B(\lambda, d)$. Applying (3.3), we obtain for every $\mu \in B(\lambda, d)$, $g \in \mathcal{O}_{M}(\mathbb{R}), v \in \mathcal{S}(\mathbb{R})$ and $n=0, \ldots, l, x \in \mathbb{R}$ that

$$
\begin{aligned}
|v(x)|\left|\left(M_{\frac{1}{\mu-h}} g(x)\right)^{(n)}\right| & \leq \sum_{i=0}^{n}\left(\begin{array}{l}
n \\
i
\end{array}\right)\left|\left(\frac{1}{\mu-h(x)}\right)^{(n-i)}\right|\left|v(x) g^{(i)}(x)\right| \\
& \leq \sum_{i=0}^{n}\left(\begin{array}{l}
n \\
i
\end{array}\right) \frac{C^{l} D}{d^{l+1}}\left(1+x^{2}\right)^{j l}\left|v(x) g^{(i)}(x)\right| \leq \frac{(2 C)^{l} D}{d^{l+1}} p_{l, w}(g),
\end{aligned}
$$

where $w(x):=\left(1+x^{2}\right)^{j l} v(x)$ for $x \in \mathbb{R}$ and hence, $w \in \mathcal{S}(\mathbb{R})$. Therefore, we get for every $\mu \in B(\lambda, d)$ and $g \in \mathcal{O}_{M}(\mathbb{R})$ that

$$
p_{l, v}\left(M_{\frac{1}{\mu-h}} g\right) \leq \frac{(2 C)^{l} D}{d^{l+1}} p_{l, w}(g)
$$

Since $l \in \mathbb{N}$ and $v \in \mathcal{S}(\mathbb{R})$ are arbitrary, this means that the set $\left\{M_{\frac{1}{\mu-h}}: \mu \in B(\lambda, d)\right\}$ is equicontinuous in $\mathcal{L}\left(\mathcal{O}_{M}(\mathbb{R})\right)$.

Remark 3.5 The description of $\sigma\left(M_{h}\right)$ given in (3.1) cannot be improved. Indeed, for the function $h(x):=e^{-x^{2}}$ for $x \in \mathbb{R}$ we have $0 \notin \operatorname{Im} h$ as $\left.\left.\operatorname{Im} h=\right] 0,1\right]$. But, $0 \notin \rho\left(M_{h}\right)$, see Remark 3.3. So, by Theorem $3.4 \sigma^{*}\left(M_{h}\right)=\sigma\left(M_{h}\right)=[0,1]$.

On the other hand, for the function $k(x)=\frac{1}{1+x^{2}}$ for $x \in \mathbb{R}$ we have that $0 \notin \operatorname{Im} k=$ ]0, 1] and $\frac{1}{k(x)}=1+x^{2} \in \mathcal{O}_{M}(\mathbb{R})$. By Proposition 3.2 the operator $M_{k}: \mathcal{S}(\mathbb{R}) \rightarrow$ $\mathcal{S}(\mathbb{R})$ is surjective and hence, $M_{k}: \mathcal{S}(\mathbb{R}) \rightarrow \mathcal{S}(\mathbb{R})$ is also injective. Thus, $M_{k}$ is a topological isomorphism from $\mathcal{S}(\mathbb{R})$ onto itself and so $0 \in \rho\left(M_{k}\right)$. So, by Theorem 3.4 we get that $\left.\left.\sigma\left(M_{k}\right)=\right] 0,1\right] \varsubsetneqq \sigma^{*}\left(M_{k}\right)=[0,1]$.

As a consequence of Theorem 3.4 we can determine the spectra of the convolution operators $C_{T}$ acting on $\mathcal{S}(\mathbb{R})$ (and on $\mathcal{O}_{C}(\mathbb{R})$ ) via the properties of the Fourier transform. 
Theorem 3.6 Let $T \in \mathcal{O}_{C}^{\prime}(\mathbb{R})$. Then the spectra of the convolution operator $C_{T}$ acting either on $\mathcal{S}(\mathbb{R})$ or on $\mathcal{O}_{C}(\mathbb{R})$ are given by:

$$
\operatorname{Im} \mathcal{F}(T) \subseteq \sigma\left(C_{T}\right) \subseteq \overline{\operatorname{Im} \mathcal{F}(T),} \sigma^{*}\left(C_{T}\right)=\overline{\operatorname{Im} \mathcal{F}(T)}
$$

Moreover, the point spectrum of the convolution operator $C_{T}$ acting on $\mathcal{S}(\mathbb{R})$ is given by:

$$
\sigma_{p}\left(C_{T}\right)=\left\{\lambda \in \mathbb{C}:(\mathcal{F}(T))^{-1}(\lambda) \text { has a non empty interior }\right\} \subseteq \operatorname{Im} \mathcal{F}(T)
$$

\section{Ergodic properties of multiplication and convolution operators on $\mathcal{S}(\mathbb{R})$}

The aim of this section is to investigate the ergodic properties of the multiplication operators $M_{h}$, for $h \in \mathcal{O}_{M}(\mathbb{R})$, and the convolution operators $C_{T}$, for $T \in \mathcal{O}_{C}^{\prime}(\mathbb{R})$, when acting on the space $\mathcal{S}(\mathbb{R})$.

So, we first observe that if $f \in \mathcal{S}(\mathbb{R})$ (or $f \in \mathcal{O}_{M}(\mathbb{R})$ ) and $n \in \mathbb{N}$,

$$
M_{h}^{n} f(x)=h^{n}(x) f(x), \quad x \in \mathbb{R} .
$$

Therefore, if $f \in \mathcal{S}(\mathbb{R})$ (or $f \in \mathcal{O}_{M}(\mathbb{R})$ ) and $n \in \mathbb{N}$, the $n$-th Cesàro mean of $M_{h}$ is given by

$$
\left(M_{h}\right)_{[n]} f(x)=\frac{1}{n} \sum_{m=1}^{n} M_{h}^{m} f(x)=\frac{1}{n} \sum_{m=1}^{n} h^{m}(x) f(x)=\frac{f(x)}{n} \sum_{m=1}^{n} h^{m}(x), \quad x \in \mathbb{R} .
$$

If we set $h_{[n]}:=\frac{1}{n} \sum_{m=1}^{n} h^{m}$ for $n \in \mathbb{N}$, then $\left(M_{h}\right)_{[n]} f=h_{[n]} f$ for any $f \in \mathcal{S}(\mathbb{R})$ (or $f \in \mathcal{O}_{M}(\mathbb{R})$ ).

We point out that if $h \in C^{\infty}(\mathbb{R})$, then $M_{h} \in \mathcal{L}\left(C^{\infty}(\mathbb{R})\right)$ and $M_{h}^{n} f=h^{n} f$ $\left(\left(M_{h}\right)_{[n]} f=h_{[n]} f\right)$ for $f \in C^{\infty}(\mathbb{R})$ and $n \in \mathbb{N}$. In this case the following result holds.

Proposition 4.1 Let $h \in C^{\infty}(\mathbb{R})$. Then the following properties are satisfied.

(1) $M_{h}: C^{\infty}(\mathbb{R}) \rightarrow C^{\infty}(\mathbb{R})$ is power bounded if and only if $\left\{h^{n}\right\}_{n \in \mathbb{N}}$ is a bounded sequence in $C^{\infty}(\mathbb{R})$.

(2) $M_{h}: C^{\infty}(\mathbb{R}) \rightarrow C^{\infty}(\mathbb{R})$ is mean ergodic if and only if $\left\{h_{[n]}\right\}_{n \in \mathbb{N}}$ is a convergent sequence in $C^{\infty}(\mathbb{R})$.

Proof (1) If $M_{h}$ is power bounded, then the sequence $\left\{M_{h}^{n} \mathbf{1}\right\}_{n \in \mathbb{N}}=\left\{h^{n}\right\}_{n \in \mathbb{N}}$ is necessarily bounded in $C^{\infty}(\mathbb{R})$.

Conversely, fixed a compact subset $K$ of $\mathbb{R}$ and an integer $m \in \mathbb{N}$, there exists $C_{K, m}>0$ such that

$$
\sup _{n \in \mathbb{N}} \sup _{x \in K} \sup _{0 \leq i \leq m}\left|\left(h^{n}(x)\right)^{(i)}\right| \leq C_{K, m} .
$$


This implies for every $n \in \mathbb{N}$ that

$$
\begin{aligned}
\sup _{x \in K} \sup _{0 \leq i \leq m}\left|\left(M_{h}^{n} f\right)^{(i)}(x)\right| & \leq \sup _{x \in K} \sup _{0 \leq i \leq m} \sum_{j=0}^{i}\left(\begin{array}{c}
i \\
j
\end{array}\right)\left|\left(h^{n}(x)\right)^{i-j}\right|\left|f^{(j)}(x)\right| \\
& \leq 2^{m} C_{K, m} \sup _{x \in K} \sup _{0 \leq i \leq m}\left|f^{(i)}(x)\right| .
\end{aligned}
$$

Since $K$ and $m$ are arbitrary, this means that the operator $M_{h}$ is power bounded.

(2) follows in a similar way.

Proposition 4.1 suggests that similar characterizations of the power boundedness and of the mean ergodicity might hold for multiplication operators acting on $\mathcal{S}(\mathbb{R})$. To see this, we begin by stating and proving the following fact.

Lemma 4.2 Let $\left\{h_{n}\right\}_{n \in \mathbb{N}} \subseteq \mathcal{O}_{M}(\mathbb{R})$. If $\left\{M_{h_{n}}\right\}_{n \in \mathbb{N}} \subseteq \mathcal{L}(\mathcal{S}(\mathbb{R}))$ is an equicontinuous sequence, then the sequence $\left\{h_{n}\right\}_{n \in \mathbb{N}}$ is bounded in $\mathcal{O}_{M}(\mathbb{R})$.

Proof Since $\mathcal{S}(\mathbb{R})$ is continuously included in $C^{\infty}(\mathbb{R})$, the assumption implies that $\left\{M_{h_{n}} f\right\}_{n \in \mathbb{N}}$ is a bounded subset of $C^{\infty}(\mathbb{R})$ whenever $f \in \mathcal{S}(\mathbb{R})$. This necessarily yields that $\left\{h_{n}\right\}_{n \in \mathbb{N}}$ is a bounded sequence in $C^{\infty}(\mathbb{R})$. Indeed, fixed a compact subset $K$ of $\mathbb{R}$ and choosen $f \in \mathcal{D}(\mathbb{R})$ such that $f(x)=1$ for every $x \in K$, we obtain for every $n \in \mathbb{N}$ and $m \in \mathbb{N}$ that

$$
\sup _{x \in K} \sup _{0 \leq i \leq m}\left|\left(h_{n}(x)\right)^{(i)}\right|=\sup _{x \in K} \sup _{0 \leq i \leq m}\left|\left(h_{n}(x) f(x)\right)^{(i)}\right| \leq C,
$$

where $C:=\sup _{n \in \mathbb{N}} \sup _{x \in K} \sup _{0 \leq i \leq m}\left|\left(h_{n}(x) f(x)\right)^{(i)}\right|<+\infty$ as $f \in \mathcal{S}(\mathbb{R})$. Since $K$ is an arbitrary compact subset of $\mathbb{R}$, this means that $\left\{h_{n}\right\}_{n \in \mathbb{N}}$ is a bounded sequence of $C^{\infty}(\mathbb{R})$.

The sequence $\left\{h_{n}\right\}_{n \in \mathbb{N}}$ is also bounded in $\mathcal{O}_{M}(\mathbb{R})$. Otherwise, there exists $m \in \mathbb{N}$ such that the sequence $\left\{h_{n}\right\}_{n \in \mathbb{N}}$ is not bounded in $\cup_{k=1}^{\infty}\left\{f \in C^{\infty}(\mathbb{R}):|f|_{m, k}=\right.$ $\left.\sup _{x \in \mathbb{R}} \sup _{0 \leq i \leq m}\left(1+x^{2}\right)^{-k}\left|f^{(i)}(x)\right|<\infty\right\}$. So, for every $k \in \mathbb{N}$ we have

$$
\sup _{n \in \mathbb{N}}\left|h_{n}\right|_{m, k}=\infty
$$

Consequently, taking into account that $\left\{h_{n}\right\}_{n \in \mathbb{N}}$ is a bounded sequence of $C^{\infty}(\mathbb{R})$, we can find a sequence $\left\{x_{k}\right\}_{k \in \mathbb{N}} \subseteq \mathbb{R}$ with $\left|x_{k}\right|>\left|x_{k-1}\right|+2$, for $k \geq 2$, an increasing sequence $\left\{n_{k}\right\}_{k \in \mathbb{N}}$ of positive integers and a sequence $\left\{i_{k}\right\}_{k \in \mathbb{N}} \subseteq\{0,1, \ldots, m\}$ such that

$$
\left(1+x_{k}^{2}\right)^{-k}\left|\left(h_{n_{k}}\right)^{\left(i_{k}\right)}\left(x_{k}\right)\right|>k, \quad k \in \mathbb{N} .
$$

Since the set $\{0,1, \ldots, m\}$ is finite, we can assume without loss of generality (indeed, it suffices to pass to a subsequence) that (4.1) is valid for every $k \in \mathbb{N}$ with the same index $i$ in $\{0,1, \ldots, m\}$. Now, let $\rho \in \mathcal{D}(\mathbb{R})$ such that supp $\rho \subseteq]-2,2[$ and $\rho(x)=1$ for $x \in[-1,1]$. Then the function 


$$
\rho(x)=\sum_{k=1}^{\infty} \frac{\rho\left(x-x_{k}\right)}{\left(1+x_{k}^{2}\right)^{k}}, \quad x \in \mathbb{R},
$$

belongs to $\mathcal{S}(\mathbb{R})$, see, f.i., [16, Proposition 4, Chap. $4 \S 11]$. Since $\left\{M_{h_{n}}\right\}_{n \in \mathbb{N}}$ is an equicontinuous sequence in $\mathcal{L}(\mathcal{S}(\mathbb{R}))$, the sequence $\left\{M_{h_{n}} \rho\right\}_{n \in \mathbb{N}}=\left\{h_{n} \rho\right\}_{n \in \mathbb{N}}$ is bounded in $\mathcal{S}(\mathbb{R})$. This implies that

$$
\sup _{n \in \mathbb{N}} \sup _{x \in \mathbb{R}}\left|\left(h_{n}(x) \rho(x)\right)^{(i)}\right|=: C<\infty .
$$

But, by (4.1) we get

$\sup _{k \in \mathbb{N}} \sup _{\left|x-x_{k}\right|<1}\left|\left(h_{n_{k}}(x) \rho(x)\right)^{(i)}\right|=\sup _{k \in \mathbb{N}\left|x-x_{k}\right|<1} \sup _{\mid<}\left|\frac{\left(h_{n_{k}}\right)^{(i)}(x)}{\left(1+x_{k}^{2}\right)^{k}}\right| \geq \sup _{k \in \mathbb{N}}\left|\frac{\left(h_{n_{k}}\right)^{(i)}\left(x_{k}\right)}{\left(1+x_{k}^{2}\right)^{k}}\right|=+\infty$.

This is a contradiction with (4.2).

The main results of this section are the following.

Theorem 4.3 Let $h \in \mathcal{O}_{M}(\mathbb{R})$. Then the following properties are equivalent.

(1) $M_{h}$ is power bounded on $\mathcal{S}(\mathbb{R})$.

(1)' $\mathcal{M}_{h}$ is power bounded on $\mathcal{S}^{\prime}(\mathbb{R})$.

(2) $M_{h}$ is power bounded on $\mathcal{O}_{M}(\mathbb{R})$.

(2)' $\mathcal{M}_{h}$ is power bounded on $\mathcal{O}_{M}^{\prime}(\mathbb{R})$.

(3) The sequence $\left\{h^{n}\right\}_{n \in \mathbb{N}}$ is bounded in $\mathcal{O}_{M}(\mathbb{R})$.

Proof $(1) \Leftrightarrow(1)$ ' follows from [18, (6), p.138], after having observed that $\mathcal{M}_{h}=M_{h}^{\prime}$ and $\mathcal{S}(\mathbb{R})$ is a reflexive lcHs.

$(2) \Leftrightarrow(2)$ ' follows by the same arguments above.

$(1) \Rightarrow(2)$. Since $M_{h}$ is power bounded on $\mathcal{S}(\mathbb{R})$, i.e., $\left\{M_{h}^{n}\right\}_{n \in \mathbb{N}}=\left\{M_{h^{n}}\right\}_{n \in \mathbb{N}} \subseteq$ $\mathcal{L}(\mathcal{S}(\mathbb{R}))$ is an equicontinuous sequence, we can apply Lemma 4.2 to conclude that $\left\{h^{n}\right\}_{n \in \mathbb{N}}$ is a bounded sequence in $\mathcal{O}_{M}(\mathbb{R})$.

Since $\left\{h^{n}\right\}_{n \in \mathbb{N}}$ is a bounded sequence in $\mathcal{O}_{M}(\mathbb{R})$, for each $v \in \mathcal{S}(\mathbb{R})$ and $m \in \mathbb{N}$ there exists $C_{m, v}>0$ such that

$$
\sup _{n \in \mathbb{N}} p_{m, v}\left(h^{n}\right) \leq C_{m, v}
$$

This together with (2.1) clearly implies that $\left\{M_{h}^{n}\right\}_{n \in \mathbb{N}}$ is an equicontinuous set in $\mathcal{L}\left(\mathcal{O}_{M}(\mathbb{R})\right)$, i.e., $M_{h}$ is power bounded when it acting on $\mathcal{O}_{M}(\mathbb{R})$. Indeed, fixed $v \in$ $\mathcal{S}(\mathbb{R})$ and $m \in \mathbb{N}$ and choosen $v_{1}, v_{2} \in \mathcal{S}(\mathbb{R})$ and $m^{\prime} \in \mathbb{N}$ as in (2.1), we have for every $n \in \mathbb{N}$ and $f \in \mathcal{O}_{M}(\mathbb{R})$ that

$$
p_{m, v}\left(M_{h}^{n} f\right)=p_{m, v}\left(h^{n} f\right) \leq p_{m^{\prime}, v_{1}}\left(h^{n}\right) p_{m^{\prime}, v_{2}}(f) \leq C_{m^{\prime}, v_{1}} p_{m^{\prime}, v_{2}}(f) .
$$

$(2) \Rightarrow(3)$. The operator $M_{h}$ is power bounded on $\mathcal{O}_{M}(\mathbb{R})$. Accordingly, for the constant function $\mathbf{1}$ which belongs to $\mathcal{O}_{M}(\mathbb{R})$, the sequence $\left\{M_{h}^{n} \mathbf{1}\right\}_{n \in \mathbb{N}}=\left\{h^{n}\right\}_{n \in \mathbb{N}}$ is bounded in $\mathcal{O}_{M}(\mathbb{R})$. 
(3) $\Rightarrow(1)$. Let $m \in \mathbb{N}$ be fixed. Since $\left\{h^{n}\right\}_{n \in \mathbb{N}}$ is a bounded sequence in $\mathcal{O}_{M}(\mathbb{R})$ and $\mathcal{O}_{M}(\mathbb{R})$ is the projective limit of the regular(LB)-spaces $\left\{\cup_{k=1}^{\infty}\left\{f \in C^{\infty}(\mathbb{R}):|f|_{l, k}=\right.\right.$ $\left.\left.\sup _{x \in \mathbb{R}} \sup _{0 \leq i \leq l}\left(1+x^{2}\right)^{-k}\left|f^{(i)}(x)\right|<\infty\right\}\right\}_{l \in \mathbb{N}}$, it is also a bounded sequence in $\cup_{k=1}^{\infty}\left\{f \in C^{\infty}(\mathbb{R}):|f|_{m, k}=\sup _{x \in \mathbb{R}} \sup _{0 \leq i \leq m}\left(1+x^{2}\right)^{-k}\left|f^{(i)}(x)\right|<\infty\right\}$ and hence, there exists $k \in \mathbb{N}$ such that

$$
\sup _{n \in \mathbb{N}}\left|h^{n}\right|_{m, k}=: C<\infty .
$$

Therefore, we obtain for every $n \in \mathbb{N}$ and $f \in \mathcal{S}(\mathbb{R})$ that

$$
\begin{aligned}
\left\|M_{h}^{n} f\right\|_{m} & =\sup _{x \in \mathbb{R}} \sup _{0 \leq i \leq m}\left(1+x^{2}\right)^{m}\left|\left(h^{n}(x) f(x)\right)^{(i)}\right| \\
& \leq \sup _{x \in \mathbb{R}} \sup _{0 \leq i \leq m}\left(1+x^{2}\right)^{m} \sum_{j=0}^{i}\left(\begin{array}{l}
i \\
j
\end{array}\right)\left|\left(h^{n}(x)\right)^{(j)} f^{(i-j)}(x)\right| \\
& \leq \sup _{x \in \mathbb{R}} \sup _{0 \leq i \leq m}\left(1+x^{2}\right)^{m+k} \sum_{j=0}^{i}\left(\begin{array}{l}
i \\
j
\end{array}\right)\left(1+x^{2}\right)^{-k}\left|\left(h^{n}(x)\right)^{(j)}\right|\left|f^{(i-j)}(x)\right| \\
& \leq 2^{m} C|| f \|_{m+k} .
\end{aligned}
$$

This means that $M_{h}$ is power bounded on $\mathcal{S}(\mathbb{R})$.

Theorem 4.4 Let $h \in \mathcal{O}_{M}(\mathbb{R})$. Then the following properties are equivalent.

(1) $M_{h}$ is (uniformly) mean ergodic on $\mathcal{S}(\mathbb{R})$.

(1)' $\mathcal{M}_{h}$ is (uniformly) mean ergodic on $\mathcal{S}^{\prime}(\mathbb{R})$.

(2) $M_{h}$ is (uniformly) mean ergodic on $\mathcal{O}_{M}(\mathbb{R})$.

(2)' $\mathcal{M}_{h}$ is (uniformly) mean ergodic on $\mathcal{O}_{M}^{\prime}(\mathbb{R})$.

(3) The sequence $\left\{h_{[n]}\right\}_{n \in \mathbb{N}}$ converges to some $g$ in $\mathcal{O}_{M}(\mathbb{R})$.

Proof (1) $\Leftrightarrow(1)$ ' follows from [3, Lemma 2.1], after having observed that $\mathcal{M}_{h}=M_{h}^{\prime}$ and $\mathcal{S}(\mathbb{R})$ is a reflexive lcHs (and hence, $\mathcal{S}^{\prime}(\mathbb{R})$ is a barrelled reflexive lcHs).

$(2) \Leftrightarrow(2)$ ' follows as above.

We now establish the equivalence between properties (2) and (3).

$(2) \Rightarrow(3)$. The operator $M_{h}$ is mean ergodic on $\mathcal{O}_{M}(\mathbb{R})$. Accordingly, there exists $P \in \mathcal{L}\left(\mathcal{O}_{M}(\mathbb{R})\right)$ such that $\left(M_{h}\right)_{[n]} \rightarrow P$ in $\mathcal{L}_{S}\left(\mathcal{O}_{M}(\mathbb{R})\right)$ as $n \rightarrow \infty$. Since the constant function 1 belongs to $\mathcal{O}_{M}(\mathbb{R})$, it follows that $\left(M_{h}\right)_{[n]} \mathbf{1}=h_{[n]} \rightarrow P \mathbf{1}=: g$ in $\mathcal{O}_{M}(\mathbb{R})$.

$(3) \Rightarrow(2)$. Fixed $m \in \mathbb{N}$ and $v \in \mathcal{S}(\mathbb{R})$ and choosen $v_{1}, v_{2} \in \mathcal{S}(\mathbb{R})$ and $m^{\prime} \in \mathbb{N}$ as in (2.1), we obtain for every $f \in \mathcal{O}_{M}(\mathbb{R})$ and $n \in \mathbb{N}$ that

$$
\begin{aligned}
p_{m, v}\left(\left(M_{h}\right)_{[n]} f-f g\right) & =p_{m, v}\left(h_{[n]} f-f g\right)=p_{m, v}\left(\left(h_{[n]}-g\right) f\right) \\
& \leq p_{m^{\prime}, v_{1}}\left(h_{[n]}-g\right) p_{m^{\prime}, v_{2}}(f) .
\end{aligned}
$$

Since $h_{[n]} \rightarrow g$ in $\mathcal{O}_{M}(\mathbb{R})$ and $m \in \mathbb{N}, v \in \mathcal{S}(\mathbb{R})$ and $f \in \mathcal{O}_{M}(\mathbb{R})$ are arbitrary, from (4.4) it follows that $\left(M_{h}\right)_{[n]} f \rightarrow f g$ in $\mathcal{O}_{M}(\mathbb{R})$ as $n \rightarrow \infty$. This means that $M_{h}$ is 
mean ergodic on $\mathcal{O}_{M}(\mathbb{R})$ and hence, uniformly mean ergodic, being $\mathcal{O}_{M}(\mathbb{R})$ a Montel lcHs.

Actually, from (4.4) it directly follows that $M_{h}$ is uniformly mean ergodic on $\mathcal{O}_{M}(\mathbb{R})$. Indeed, for a fixed bounded subset $B$ of $\mathcal{O}_{M}(\mathbb{R})$, we obtain by (4.4) for every $n \in \mathbb{N}$ that

$$
\sup _{f \in B} p_{m, v}\left(\left(M_{h}\right)_{[n]} f-f g\right) \leq p_{m^{\prime}, v_{1}}\left(h_{[n]}-g\right) \sup _{f \in B} p_{m^{\prime}, v_{2}}(f)
$$

where $\sup _{f \in B} p_{m^{\prime}, v_{2}}(f)<\infty$ and $p_{m^{\prime}, v_{1}}\left(h_{[n]}-g\right) \rightarrow 0$ as $n \rightarrow \infty$.

(3) $\Rightarrow(1)$. Let $f \in \mathcal{S}(\mathbb{R})$ and $m \in \mathbb{N}$ be fixed. Then there exists $v \in \mathcal{S}(\mathbb{R})$ such that

$$
\sup _{0 \leq j \leq m}\left(1+x^{2}\right)^{m}\left|f^{(j)}(x)\right| \leq v(x)
$$

for every $x \in \mathbb{R}$ (see [9, Lemma 3.6, p. 127]). Thus, we obtain for every $n \in \mathbb{N}$ that

$$
\begin{aligned}
& \left\|\left(M_{h}\right)_{[n]} f-g f\right\|_{m}=\sup _{x \in \mathbb{R}} \sup _{0 \leq i \leq m}\left(1+x^{2}\right)^{m}\left|\left[\left(h_{[n]}(x)-g(x)\right) f(x)\right]^{(i)}\right| \\
& \leq \sup _{x \in \mathbb{R}} \sup _{0 \leq i \leq m}\left(1+x^{2}\right)^{m} \sum_{j=0}^{i}\left(\begin{array}{c}
i \\
j
\end{array}\right)\left|\left(h_{[n]}(x)-g(x)\right)^{(j)}\right|\left|f^{(j-i)}(x)\right| \\
& \leq 2^{m} \sup _{x \in \mathbb{R}} \sup _{0 \leq i \leq m} v(x)\left|\left(h_{[n]}(x)-g(x)\right)^{(i)}\right|=p_{m, v}\left(h_{[n]}-g\right) .
\end{aligned}
$$

Since $h_{[n]} \rightarrow g$ in $\mathcal{O}_{M}(\mathbb{R})$ and $m \in \mathbb{N}$ is arbitrary, this implies that $\left(M_{h}\right)_{[n]} f \rightarrow g f$ in $\mathcal{S}(\mathbb{R})$ as $n \rightarrow \infty$. As $f \in \mathcal{S}(\mathbb{R})$ is also arbitary, we can conclude that $M_{h}$ is mean ergodic when it acts on $\mathcal{S}(\mathbb{R})$ and hence, uniformly mean ergodic, being $\mathcal{S}(\mathbb{R})$ a Montel Fréchet space.

To complete the proof, it remains to show that $(1) \Rightarrow(2)$.

$(1) \Rightarrow(2)$. Since $\mathcal{S}(\mathbb{R})$ is continuously included in $C^{\infty}(\mathbb{R})$, the assumption implies that $\left(M_{h}\right)_{[n]} f \rightarrow P f$ in $C^{\infty}(\mathbb{R})$ too, as $n \rightarrow \infty$, whenever $f \in \mathcal{S}(\mathbb{R})$. This necessarily implies that $\left\{h_{[n]}\right\}_{n \in \mathbb{N}}$ converges to some $g$ in $C^{\infty}(\mathbb{R})$. Indeed, fixed a compact subset $K$ of $\mathbb{R}$ and choosen $f \in \mathcal{D}(\mathbb{R})$ such that $f(x)=1$ for every $x \in K$, we have for every $l, m, n \in \mathbb{N}$ that

$$
\begin{aligned}
& \sup _{x \in K} \sup _{0 \leq i \leq l}\left|\left(h_{[n]}(x)-h_{[m]}(x)\right)^{(i)}\right|=\sup _{x \in K} \sup _{0 \leq i \leq l}\left|\left[\left(h_{[n]}(x)-h_{[m]}(x)\right) f(x)\right]^{(i)}\right| \\
& \leq \sup _{x \in K} \sup _{0 \leq i \leq l}\left|\left(h_{[m]}(x) f(x)-P f(x)\right)^{(i)}\right|+\sup _{x \in K} \sup _{0 \leq i \leq l}\left|\left(h_{[n]}(x) f(x)-P f(x)\right)^{(i)}\right| \\
& =: a_{m}+a_{n} .
\end{aligned}
$$

Since $\left(M_{h}\right)_{[n]} f=h_{[n]} f \rightarrow P f$ in $C^{\infty}(\mathbb{R})$ as $n \rightarrow \infty$, we get that $a_{m}+a_{n} \rightarrow 0$ as $m, n \rightarrow \infty$. Hence, it follows via (4.6) that $\sup _{x \in K} \sup _{0 \leq i \leq l}\left|\left(h_{[n]}(x)-h_{[m]}(x)\right)^{(i)}\right| \rightarrow$ 0 as $m, n \rightarrow \infty$. So, as $K$ is an arbitrary compact subset of $\mathbb{R}$, this shows that $\left\{h_{[n]}\right\}_{n \in \mathbb{N}}$ is a Cauchy sequence in $C^{\infty}(\mathbb{R})$. Accordingly, $h_{[n]} \rightarrow g$ in $C^{\infty}(\mathbb{R})$ as $n \rightarrow \infty$. 
The facts that $\left(M_{h}\right)_{[n]} f=h_{[n]} f \rightarrow P f$ and $\left(M_{h}\right)_{[n]} f=h_{[n]} f \rightarrow g f$ in $C^{\infty}(\mathbb{R})$ as $n \rightarrow \infty$ for every $f \in \mathcal{S}(\mathbb{R})$, imply that $g f=P f \in \mathcal{S}(\mathbb{R})$ for every $f \in \mathcal{S}(\mathbb{R})$. Therefore, $g \in \mathcal{O}_{M}(\mathbb{R})$.

By Lemma 4.2 the assumption also implies that $\left\{h_{[n]}\right\}_{n \in \mathbb{N}}$ is a bounded sequence in $\mathcal{O}_{M}(\mathbb{R})$. Indeed, $\left\{\left(M_{h}\right)_{[n]}\right\}_{n \in \mathbb{N}}=\left\{M_{h_{[n]}}\right\}_{n \in \mathbb{N}}$ is an equicontinuous sequence in $\mathcal{L}(\mathcal{S}(\mathbb{R}))$, being a convergent sequence in $\mathcal{L}_{S}(\mathcal{S}(\mathbb{R}))$. Accordingly, for each $v \in \mathcal{S}(\mathbb{R})$ and $m \in \mathbb{N}$ there exists $C_{m, v}>0$ such that

$$
\sup _{n \in \mathbb{N}} p_{m, v}\left(h_{[n]}\right) \leq C_{m, v} .
$$

This together with (2.1) clearly implies that $\left\{\left(M_{h}\right)_{[n]}\right\}_{n \in \mathbb{N}}$ is an equicontinuous set in $\mathcal{L}\left(\mathcal{O}_{M}(\mathbb{R})\right)$. Indeed, fixed $v \in \mathcal{S}(\mathbb{R})$ and $m \in \mathbb{N}$ and choosen $v_{1}, v_{2} \in \mathcal{S}(\mathbb{R})$ and $m^{\prime} \in \mathbb{N}$ as in (2.1), we have for every $n \in \mathbb{N}$ and $f \in \mathcal{O}_{M}(\mathbb{R})$ that

$$
p_{m, v}\left(\left(M_{h}\right)_{[n]} f\right)=p_{m, v}\left(h_{[n]} f\right) \leq p_{m^{\prime}, v_{1}}\left(h_{[n]}\right) p_{m^{\prime}, v_{2}}(f) \leq C_{m^{\prime}, v_{1}} p_{m^{\prime}, v_{2}}(f) .
$$

Since $\left\{\left(M_{h}\right)_{[n]}\right\}_{n \in \mathbb{N}}$ is an equicontinuous set in $\mathcal{L}\left(\mathcal{O}_{M}(\mathbb{R})\right)$ and $\left(M_{h}\right)_{[n]} f \rightarrow \operatorname{Pf}(=$ $g f$ ) in $\mathcal{S}(\mathbb{R})$ as $n \rightarrow \infty$ (and hence in $\mathcal{O}_{M}(\mathbb{R})$ ), and $\mathcal{S}(\mathbb{R})$ is a dense subspace of $\mathcal{O}_{M}(\mathbb{R})$, we deduce that $\left(M_{h}\right)_{[n]} f \rightarrow P f(=g f)$ in $\mathcal{O}_{M}(\mathbb{R})$ as $n \rightarrow \infty$, for every $f \in \mathcal{O}_{M}(\mathbb{R})$. So, $M_{h}$ is mean ergodic on $\mathcal{O}_{M}(\mathbb{R})$ and hence, uniformly mean ergodic, being $\mathcal{O}_{M}(\mathbb{R})$ a Montel lcHs.

We now point out the following fact which could be useful for applications.

Proposition 4.5 Let $h \in \mathcal{O}_{M}(\mathbb{R})$ with $h \neq 1$. If the multiplication operator $M_{h}: \mathcal{S}(\mathbb{R}) \rightarrow \mathcal{S}(\mathbb{R})$ is mean ergodic, then $\|h\|_{0}=\sup _{x \in \mathbb{R}}|h(x)| \leq 1$ and $h^{-1}(1)$ is an empty subset of $\mathbb{R}$. Moreover, the operator $P=\lim _{n \rightarrow \infty}\left(M_{h}\right)_{[n]}=0$ and $\mathcal{S}(\mathbb{R})=\overline{\operatorname{Im} M_{1-h}}$.

Proof The assumption implies that $\frac{M_{h}^{n}}{n} \rightarrow 0$ in $\mathcal{L}_{S}(\mathcal{S}(\mathbb{R}))$ and hence, by Proposition 5.1, we have $\sigma\left(M_{h}\right) \subseteq \overline{\mathbb{D}}$. On the other hand, by Theorem 3.4, Im $h \subseteq \sigma\left(M_{h}\right)$. So, it follows that $\|h\|_{0} \leq 1$.

Suppose that $h^{-1}(1) \neq \emptyset$. Since $\|h\|_{0} \leq 1$ and

$$
h_{[n]}(x)=\frac{1}{n} \sum_{m=1}^{n} h^{m}(x)=\left\{\begin{array}{cc}
\frac{h(x)}{n} \frac{1-h^{n}(x)}{1-h(x)}, & \text { if } h(x) \neq 1, \\
1, & \text { if } h(x)=1,
\end{array}\right.
$$

for every $n \in \mathbb{N}$, it follows that

$$
\lim _{n \rightarrow \infty} h_{[n]}(x)=\left\{\begin{array}{l}
0, \text { if } h(x) \neq 1, \\
1, \text { if } h(x)=1 .
\end{array}\right.
$$

This is a contradiction with the fact that by Theorem 4.4 the sequence $\left\{h_{[n]}\right\}_{n \in \mathbb{N}}$ converges to some function $g$ in $\mathcal{O}_{M}(\mathbb{R})$, where $g$ is obviuosly a continuous function on $\mathbb{R}$. 
Since $h^{-1}(1)$ is an empty subset of $\mathbb{R}$, the sequence $\left\{h_{[n]}\right\}_{n \in \mathbb{N}}$ necessarily converges to 0 in $\mathcal{O}_{M}(\mathbb{R})$ and hence, $P=0$. Accordingly, $\mathcal{S}(\mathbb{R})=\overline{\operatorname{Im}\left(I-M_{h}\right)}=\overline{\operatorname{Im} M_{1-h}}$.

In view of Proposition 4.5 we can now collect some examples.

Example 4.6 For a fixed $n \in \mathbb{N}$, we observe that the function $f_{n}(x)=\frac{1}{(1+x)^{n}}, x \neq-1$ belongs to $C^{\infty}(\mathbb{R} \backslash\{-1\})$ and

$$
f_{n}^{(j)}(x)=\frac{(-1)^{j} n(n+1) \ldots(n+j)}{(1+x)^{n+j}}, \quad j \in \mathbb{N}, x \neq-1,
$$

as it is easy to verify.

(a) Consider the function $h(x):=\frac{1}{1+a e^{i x}}$, for $x \in \mathbb{R}$, with $|a|>2$. If we set $g(x):=a e^{i x}$ for $x \in \mathbb{R}$, then $h^{n}=f_{n} \circ g$ for every $n \in \mathbb{N}$. Therefore, by the Faà Bruno formula and (4.8), we have for every $x \in \mathbb{R}$ and $j, n \in \mathbb{N}$ that

$$
\left(h^{n}(x)\right)^{(j)}=\sum \frac{j !}{k_{1} ! \ldots k_{j} !} \frac{(-1)^{k} n(n+1) \ldots(n+k)}{\left(1+a e^{i x}\right)^{n+k}} \frac{a^{j} i^{j} e^{i j x}}{(1 !)^{k_{1}}(2 !)^{k_{2}} \ldots(j !)^{k_{j}}},
$$

where $k=k_{1}+k_{2}+\ldots+k_{j}$ and $k_{1}+2 k_{2}+\ldots+j k_{j}=j$. Accordingly, we have for every $x \in \mathbb{R}$ and $j, n \in \mathbb{N}$ that

$$
\begin{aligned}
\left|\left(h^{n}(x)\right)^{(j)}\right| & \leq \sum \frac{j !}{k_{1} ! \ldots k_{j} !} \frac{n(n+1) \ldots(n+j)}{(|a|-1)^{n+k}} \frac{|a|^{j}}{(1 !)^{k_{1}}(2 !)^{k_{2}} \ldots(j !)^{k_{j}}} \\
& =C_{j} \frac{n(n+1) \ldots(n+j)}{(|a|-1)^{n}}
\end{aligned}
$$

being $C_{j}:=|a|^{j} \sum \frac{j !}{k_{1} ! \ldots k_{j} !} \frac{1}{(1 !)^{k_{1}}(2 !)^{k_{2} \ldots(j !)^{k_{j}}}}$. It follows for every $v \in \mathcal{S}(\mathbb{R})$ and $l, n \in$ $\mathbb{N}$ that

$$
p_{l, v}\left(h^{n}\right) \leq C\|v\|_{0} \frac{n(n+1) \ldots(n+l)}{(|a|-1)^{n}} \leq C D\|v\|_{0},
$$

where $C:=\sup _{j=0, \ldots, l} C_{j}<\infty$ and $D:=\sup _{n \in \mathbb{N}} \frac{n(n+1) \ldots(n+l)}{(|a|-1)^{n}}<\infty$. This means that $\left\{h^{n}\right\}_{n \in \mathbb{N}}$ is a bounded sequence of $\mathcal{O}_{M}(\mathbb{R})$.

(b) Consider the function $h(x):=\frac{a}{1+x^{2}}$ for $x \in \mathbb{R}$, with $|a|<1$. If we set $g(x):=x^{2}$ for $x \in \mathbb{R}$, then $h^{n}=a^{n} f_{n} \circ g$ for every $n \in \mathbb{N}$. Therefore, by the Faà Bruno formula and (4.8) taking into account that $g^{(j)}(x)=0$ for $j>2$, we get for every $x \in \mathbb{R}$ and $j, n \in \mathbb{N}$ that

$$
\left(h^{n}(x)\right)^{(j)}=a^{n} \sum \frac{j !}{k_{1} ! k_{2} !} \frac{(-1)^{k} n(n+1) \ldots(n+k)}{\left(1+x^{2}\right)^{n+k}}(2 x)^{k_{1}} .
$$

Accordingly, we have for every $x \in \mathbb{R}$ and $j, n \in \mathbb{N}$ that

$$
\left|\left(h^{n}(x)\right)^{(j)}\right| \leq|a|^{n} \frac{n(n+1) \ldots(n+j)}{\left(1+x^{2}\right)^{n}} \sum \frac{j !}{k_{1} ! k_{2} !}|2 x|^{k_{1}} .
$$


If we set $q_{j}(x):=\sum \frac{j !}{k_{1} ! k_{2} !}|2 x|^{k_{1}}$ for $x \in \mathbb{R}$, it follows for every $l, n \in \mathbb{N}$ that

$$
p_{l, v}\left(h^{n}\right) \leq C|a|^{n} n(n+1) \ldots(n+l) \leq C D,
$$

where $C:=\sup _{x \in \mathbb{R}} \sup _{j=0, \ldots, l} \frac{q_{j}(x)}{\left(1+x^{2}\right)^{n}}|v(x)|<\infty$ and $D:=\sup _{n \in \mathbb{N}}|a|^{n} n(n+$ 1) $\ldots(n+l)<\infty$. This means that the sequence $\left\{h^{n}\right\}_{n \in \mathbb{N}}$ is bounded in $\mathcal{O}_{M}(\mathbb{R})$.

(c) In a similar way one shows that the sequence of the $n$-th powers of the following functions is bounded in $\mathcal{O}_{M}(\mathbb{R}): h(x):=a e^{-x^{2}}, k(x):=a e^{i x}$ and $s(x):=\frac{x}{1+x^{2}}$, for $x \in \mathbb{R}$, with $|a|<1$.

We now pass to investigate the ergodic properties of the convolution operators acting on $\mathcal{S}(\mathbb{R})$.

But first, we need to observe the following facts.

We first recall for every $n \in \mathbb{N}, f \in \mathcal{S}(\mathbb{R})$ and $S \in \mathcal{O}_{C}^{\prime}(\mathbb{R})$ that

$$
\mathcal{F}\left(C_{T}^{n}(f)\right)=\mathcal{F}(T)^{n} \hat{f}, \quad \mathcal{F}\left(\mathcal{C}_{T}^{n}(S)\right)=\mathcal{F}(T)^{n} \mathcal{F}(S)
$$

This means that

$$
\mathcal{F} \circ C_{T}^{n}=M_{\mathcal{F}(T)}^{n} \circ \mathcal{F}\left(\mathcal{F} \circ \mathcal{C}_{T}^{n}=M_{\mathcal{F}(T)}^{n} \circ \mathcal{F}, \text { resp. }\right), \quad n \in \mathbb{N} .
$$

Therefore, we have for every $n \in \mathbb{N}$ that

$$
\mathcal{F} \circ\left(C_{T}\right)_{[n]}=\left(M_{\mathcal{F}(T)}\right)_{[n]} \circ \mathcal{F} \quad\left(\mathcal{F} \circ\left(\mathcal{C}_{T}\right)_{[n]}=\left(M_{\mathcal{F}(T)}\right)_{[n]} \circ \mathcal{F}, \text { resp. }\right) .
$$

If we set

$$
(\star T)^{2}:=T \star T, \quad(\star T)^{n}:=T \star(\star T)^{n-1}(\text { for } n \geq 2)
$$

(the definition is well-posed because $T,(\star T)^{n-1} \in \mathcal{O}_{C}^{\prime}(\mathbb{R})$ and hence $(\star T)^{n}=$ $\left.T \star(\star T)^{n-1} \in \mathcal{O}_{C}^{\prime}(\mathbb{R})\right)$, then we have for every $n \in \mathbb{N}$ that

$$
\mathcal{F}\left((\star T)^{n}\right)=\mathcal{F}(T)^{n},
$$

and hence, by (4.9) that

$$
\mathcal{F}\left(\mathcal{C}_{T}^{n}(\delta)\right)=\mathcal{F}(T)^{n} \mathcal{F}(\delta)=\mathcal{F}\left((\star T)^{n}\right) \Rightarrow \mathcal{C}_{T}^{n}(\delta)=(\star T)^{n},
$$

being $\delta$ the delta of Dirac. Since $(\star T)^{n} \in \mathcal{O}_{C}^{\prime}(\mathbb{R})$ for every $n \in \mathbb{N}$, we also get for every $n \in \mathbb{N}$ that the distribution $(\star T)_{[n]}:=\frac{1}{n} \sum_{m=1}^{n}(\star T)^{m} \in \mathcal{O}_{C}^{\prime}(\mathbb{R})$ and that

$$
\left(\mathcal{C}_{T}\right)_{[n]}(\delta)=(\star T)_{[n]} .
$$

So, as a consequence of Theorems 4.3 and 4.4 we can characterize the ergodic properties of the convolution operators $C_{T}$ acting on $\mathcal{S}(\mathbb{R})$ and on $\mathcal{O}_{C}(\mathbb{R})$ via the properties of 
the Fourier transform and applying [18, (6), p.138] in the case of power boundedness ([3, Lemma 2.1] in the case of the mean ergodicity).

Proposition 4.7 Let $T \in \mathcal{O}_{C}^{\prime}(\mathbb{R})$. Then the following properties are equivalent.

(1) $C_{T}$ is power bounded on $\mathcal{S}(\mathbb{R})$.

(1)' $\mathcal{C}_{T}$ is power bounded on $\mathcal{S}^{\prime}(\mathbb{R})$.

(2) $C_{T}$ is power bounded on $\mathcal{O}_{C}(\mathbb{R})$.

(2)' $\mathcal{C}_{T}$ is power bounded on $\mathcal{O}_{C}^{\prime}(\mathbb{R})$.

(3) The sequence $\left\{(\star T)^{n}\right\}_{n \in \mathbb{N}}$ is bounded in $\mathcal{O}_{C}^{\prime}(\mathbb{R})$.

Proposition 4.8 Let $T \in \mathcal{O}_{C}^{\prime}(\mathbb{R})$. Then the following properties are equivalent.

(1) $C_{T}$ is (uniformly) mean ergodic on $\mathcal{S}(\mathbb{R})$.

(1)' $\mathcal{C}_{T}$ is (uniformly) mean ergodic on $\mathcal{S}^{\prime}(\mathbb{R})$.

(2) $C_{T}$ is (uniformly) mean ergodic on $\mathcal{O}_{C}(\mathbb{R})$.

(2)' $\mathcal{C}_{T}$ is (uniformly) mean ergodic on $\mathcal{O}_{C}^{\prime}(\mathbb{R})$.

(3) The sequence $\left\{(\star T)_{[n]}\right\}_{n \in \mathbb{N}}$ is convergent in $\mathcal{O}_{C}^{\prime}(\mathbb{R})$.

Remark 4.9 Let $T \in \mathcal{O}_{C}^{\prime}(\mathbb{R})$. If $C_{T}: \mathcal{S}(\mathbb{R}) \rightarrow \mathcal{S}(\mathbb{R})$ is either power bounded or mean ergodic, then $\|\mathcal{F}(T)\|_{0} \leq 1$ and $(\mathcal{F}(T))^{-1}(1)=\emptyset$. Moreover, $(\star T)_{[n]} \rightarrow 0$ in $\mathcal{O}_{C}^{\prime}(\mathbb{R})$ as $n \rightarrow \infty$. This easily follows from Proposition 4.5 thanks to the identity $\mathcal{F} \circ C_{T}=M_{\mathcal{F}(T)} \circ \mathcal{F}$, being $\mathcal{F}$ a topological isomorphism from $\mathcal{O}_{C}^{\prime}(\mathbb{R})$ onto $\mathcal{O}_{M}(\mathbb{R})$.

Finally, we point out that a similar characterization of the power boundedness and mean ergodicity is valid also for convolution operators acting on the strong dual $\mathcal{E}^{\prime}(\mathbb{R})$ of $C^{\infty}(\mathbb{R})$. In this case, the result does not follow as a consequence of Propositon 4.10 because the Fourier transform is not well posed on $C^{\infty}(\mathbb{R})$. But, a direct proof is needed. To state and prove the result, we observe that the same arguments as above show that if $T \in \mathcal{E}^{\prime}(\mathbb{R})$, then $(\star T)^{n}$ is well-posed and belongs to $\mathcal{E}^{\prime}(\mathbb{R})$ for any $n \in \mathbb{N}$. Hence, $(\star T)_{[n]}$ is also well posed and belongs to $\mathcal{E}^{\prime}(\mathbb{R})$ for any $n \in \mathbb{N}$.

Proposition 4.10 Let $T \in \mathcal{E}^{\prime}(\mathbb{R})$. Then the following properties are satisfied.

(1) $\mathcal{C}_{T}: \mathcal{E}^{\prime}(\mathbb{R}) \rightarrow \mathcal{E}^{\prime}(\mathbb{R})$ is power bounded if and only if $\left\{(\star T)^{n}\right\}_{n \in \mathbb{N}}$ is a bounded sequence of $\mathcal{E}^{\prime}(\mathbb{R})$.

(2) $\mathcal{C}_{T}: \mathcal{E}^{\prime}(\mathbb{R}) \rightarrow \mathcal{E}^{\prime}(\mathbb{R})$ is mean ergodic if and only if $\left\{(\star T)_{[n]}\right\}_{n \in \mathbb{N}}$ is a convergent sequence of $\mathcal{E}^{\prime}(\mathbb{R})$.

Proof (1) If $\mathcal{C}_{T}$ is power bounded, then the sequence $\left\{\mathcal{C}_{T}^{n}(\delta)\right\}_{n \in \mathbb{N}}=\left\{(\star T)^{n}\right\}_{n \in \mathbb{N}}$ is necessarily bounded in $\mathcal{E}^{\prime}(\mathbb{R})$.

Conversely, there exist a compact subset $K$ of $\mathbb{R}, m \in \mathbb{N}$ and $C_{K, m}>0$ such that

$$
\sup _{n \in \mathbb{N}} \sup _{f \in U_{K, m}}\left|\left\langle(\star T)^{n}, f\right\rangle\right| \leq C_{K, m},
$$

where $U_{K, m}:=\left\{f \in C^{\infty}(\mathbb{R}):\|f\|_{K, m}:=\sup _{x \in K} \sup _{0 \leq i \leq m}\left|f^{(i)}(x)\right| \leq 1\right\}$. So, it follows for every $i, n \in \mathbb{N}$ and $f \in C^{\infty}(\mathbb{R})$ that

$$
\left|\left((\star T)^{n} \star f\right)^{(i)}(x)\right|=\left|\left\langle(\star T)^{n},\left(\tau_{x}^{\check{\gamma}} f\right)^{(i)}\right\rangle\right| \leq C_{K, m}||\left(\tau_{x}^{\check{\gamma}} f\right)^{(i)} \|_{K, m}, \quad x \in \mathbb{R} .
$$


Therefore, for a fixed compact subset $H$ of $\mathbb{R}$ and $l \in \mathbb{N}$, this yields for every $n \in \mathbb{N}$ and $f \in C^{\infty}(\mathbb{R})$ that

$$
\sup _{x \in H} \sup _{0 \leq i \leq l}\left|\left((\star T)^{n} \star f\right)^{(i)}(x)\right| \leq C_{K, m} \sup _{z \in H-K} \sup _{0 \leq i \leq l+m}\left|f^{(i)}(z)\right| .
$$

Since $H$ and $l \in \mathbb{N}$ are arbitrary, this implies that the set $B^{*}:=\left\{(\star T)^{n} \star f: f \in B\right\}$ is bounded in $C^{\infty}(\mathbb{R})$ whenever $B$ is a bounded subset of $C^{\infty}(\mathbb{R})$. Accordingly, for a fixed bounded subset $B$ of $C^{\infty}(\mathbb{R})$ there exists a bounded subset $B^{*}$ of $C^{\infty}(\mathbb{R})$ such that every $n \in \mathbb{N}$ and $S \in \mathcal{E}^{\prime}(\mathbb{R})$ we have

$$
\sup _{f \in B}\left|\left\langle\mathcal{C}_{T}^{n}(S), f\right\rangle\right|=\sup _{f \in B}\left\langle S,(\star \check{T})^{n} \star f\right\rangle\left|\leq \sup _{g \in B^{*}}\right|\langle S, g\rangle \mid
$$

i.e., $\mathcal{C}_{T}$ is power bounded.

(2) If $\mathcal{C}_{T}$ is mean ergodic, then the sequence $\left\{\left(\mathcal{C}_{T}\right)_{[n]}(\delta)\right\}_{n \in \mathbb{N}}=\left\{(\star T)_{[n]}\right\}_{n \in \mathbb{N}}$ is convergent in $\mathcal{E}^{\prime}(\mathbb{R})$.

Conversely, there exist $T_{0} \in \mathcal{E}^{\prime}(\mathbb{R})$ (say, $T_{0}=0$ ), a compact subset $K$ of $\mathbb{R}$ and $m \in \mathbb{N}$ such that

$$
\left\|(\star T)_{[n]}\right\|_{K, m}^{\prime}:=\sup _{f \in U_{K, m}}\left|\left\langle(\star T)^{n}, f\right\rangle\right| \rightarrow 0 .
$$

So, arguing as in part (1), it follows for every $i, n \in \mathbb{N}$ and $f \in C^{\infty}(\mathbb{R})$ that

$$
\left|\left((\star T)_{[n] \star} f\right)^{(i)}(x)\right| \leq\left\|(\star T)_{[n]}\right\|_{K, m}^{\prime}\left\|\left(\tau_{x}^{\check{\tau} f}\right)^{(i)}\right\|_{K, m}, \quad x \in \mathbb{R} .
$$

Therefore, for a fixed compact subset $H$ of $\mathbb{R}$ and $l \in \mathbb{N}$, this yields for every $n \in \mathbb{N}$ and $f \in C^{\infty}(\mathbb{R})$ that

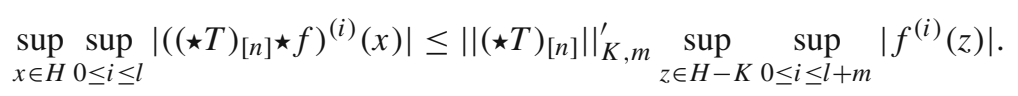

Since $H$ and $l \in \mathbb{N}$ are arbitrary, this impies that $(\star T)_{[n]} \star f \rightarrow 0$ in $C^{\infty}(\mathbb{R})$ as $n \rightarrow \infty$ whenever $f \in C^{\infty}(\mathbb{R})$.

Now, let $S \in \mathcal{E}^{\prime}(\mathbb{R})$. Then there exist a compact subset $H$ of $\mathbb{R}, l \in \mathbb{N}$ and $C>0$ such that

$$
|\langle S, f\rangle| \leq C \sup _{x \in H} \sup _{0 \leq i \leq l}\left|f^{(i)}(x)\right|
$$

for every $f \in C^{\infty}(\mathbb{R})$. So, combining (4.10) with (4.11) we obtain for every $n \in \mathbb{N}$ and $f \in C^{\infty}(\mathbb{R})$ that

$$
\left|\left\langle\left(\mathcal{C}_{T}\right)_{[n]}(S), f\right\rangle\right|=\left|\left\langle S,(\star \check{T})_{[n]} \star f\right\rangle\right| \leq C||(\star T)_{[n]} \|_{K, m}^{\prime} \sup _{z \in H-K} \sup _{0 \leq i \leq l+m}\left|f^{(i)}(z)\right| .
$$

So, for a fixed bounded subset $B$ of $C^{\infty}(\mathbb{R})$, it follows for every $n \in \mathbb{N}$ that

$$
\sup _{f \in B}\left|\left\langle\left(\mathcal{C}_{T}\right)_{[n]}(S), f\right\rangle\right| \leq C\left\|(\star T)_{[n]}\right\|_{K, m}^{\prime} \sup _{f \in B} \sup _{z \in H-K} \sup _{0 \leq i \leq l+m}\left|f^{(i)}(z)\right| .
$$


Since $\sup _{f \in B} \sup _{z \in H-K} \sup _{0 \leq i \leq l+m}\left|f^{(i)}(z)\right|<\infty$ and $\left\|(\star T)_{[n]}\right\|_{K, m}^{\prime} \rightarrow 0$ as $n \rightarrow$ $\infty$, this implies that $\sup _{f \in B}\left|\left\langle\left(\overline{\mathcal{C}}_{T}\right)_{[n]}(S), f\right\rangle\right| \rightarrow 0$ as $n \rightarrow \infty$. But $B$ is arbitrary. Then we can conclude that $\left(\mathcal{C}_{T}\right)_{[n]} \rightarrow 0$ in $\mathcal{E}^{\prime}(\mathbb{R})$ as $n \rightarrow \infty$, i.e., $\mathcal{C}_{T}$ is mean ergodic.

Remark 4.11 Since $\mathcal{O}_{M}(\mathbb{R})$ is continuously included in $C^{\infty}(\mathbb{R})$ and $\mathcal{E}^{\prime}(\mathbb{R})$ is continuously included in $\mathcal{O}_{C}^{\prime}(\mathbb{R})$, the results of this section clearly imply that:

(1) Let $h \in \mathcal{O}_{M}(\mathbb{R})$. If $M_{h}: \mathcal{S}(\mathbb{R}) \rightarrow \mathcal{S}(\mathbb{R})$ is power bounded (mean ergodic, resp.), then $M_{h}: C^{\infty}(\mathbb{R}) \rightarrow C^{\infty}(\mathbb{R})$ is power bounded (mean ergodic, resp.).

(2) Let $T \in \mathcal{E}^{\prime}(\mathbb{R})$. If $\mathcal{C}_{T}: \mathcal{E}^{\prime}(\mathbb{R}) \rightarrow \mathcal{E}^{\prime}(\mathbb{R})$ is power bounded (mean ergodic, resp.), then $\mathcal{C}_{T}: \mathcal{S}^{\prime}(\mathbb{R}) \rightarrow \mathcal{S}^{\prime}(\mathbb{R})$ is power bounded (mean ergodic, resp.).

We point out that the proof of part (1) is also given in the course of the proof of Theorems 4.3 and 4.4 .

Acknowledgements The authors would like to thank the Referee for providing many valuable comments.

Funding Open access funding provided by Universitá del Salento within the CRUI-CARE Agreement.

Open Access This article is licensed under a Creative Commons Attribution 4.0 International License, which permits use, sharing, adaptation, distribution and reproduction in any medium or format, as long as you give appropriate credit to the original author(s) and the source, provide a link to the Creative Commons licence, and indicate if changes were made. The images or other third party material in this article are included in the article's Creative Commons licence, unless indicated otherwise in a credit line to the material. If material is not included in the article's Creative Commons licence and your intended use is not permitted by statutory regulation or exceeds the permitted use, you will need to obtain permission directly from the copyright holder. To view a copy of this licence, visit http://creativecommons.org/licenses/by/4.0/.

\section{Appendix}

We establish here some general results on the spectrum of continuous linear operators acting on Fréchet spaces.

Proposition 5.1 Let $E$ be a Fréchet space and $T \in \mathcal{L}(E)$. If there exists $\lim _{n \rightarrow \infty} \frac{T^{n}}{n}=$ 0 in $\mathcal{L}_{S}(E)$, then $\sigma(T) \subseteq \overline{\mathbb{D}}$.

Proof The assumption $\tau_{s}-\lim _{n \rightarrow \infty} \frac{T^{n}}{n}=0$ implies that the set $\left\{\frac{T^{n}}{n}: n \in \mathbb{N}\right\}$ is equicontinuous in $E$. If $\left\{p_{j}\right\}_{j \in \mathbb{N}}$ is an increasing sequence of continuous seminorms generating the lc-topology of $E$, then for each $j \in \mathbb{N}$ there exist $j^{\prime} \in \mathbb{N}$ with $j^{\prime} \geq j$ and $c_{j}>0$ such that

$$
p_{j}\left(\frac{T^{n} x}{n}\right) \leq c_{j} p_{j^{\prime}}(x)
$$

for every $x \in E$ and $n \in \mathbb{N}$.

For every $j \in \mathbb{N}$ and $x \in E$ we define

$$
q_{j}(x):=\max \left\{p_{j}(x), \sup _{n \in \mathbb{N}} p_{j}\left(\frac{T^{n} x}{n}\right)\right\} .
$$


Thus, each $q_{j}$ is a seminorm on $E$ and $q_{j} \leq q_{j+1}$ for every $j \in \mathbb{N}$, as it is easy to show. On the other hand, from (5.1) it follows that

$$
p_{j}(x) \leq q_{j}(x) \leq c_{j} p_{j^{\prime}}(x)
$$

for every $j \in \mathbb{N}$ and $x \in E$. Therefore, $\left\{q_{j}\right\}_{j \in \mathbb{N}}$ is an increasing sequence of continuous seminorms generating the lc-topology of $E$. Moreover, we have for every $j, n \in \mathbb{N}$ and $x \in E$ that

$$
q_{j}\left(\frac{T^{n} x}{n}\right)=\max \left\{p_{j}\left(\frac{T^{n} x}{n}\right), \sup _{m \in \mathbb{N}} p_{j}\left(\frac{T^{n+m} x}{n m}\right)\right\} \leq 2 q_{j}(x),
$$

as $2 n m \geq n+m$ for all $n, m \in \mathbb{N}$. This yields for every $j, n \in \mathbb{N}$ and $x \in E$ that

$$
q_{j}\left(T^{n} x\right) \leq 2 n q_{j}(x)
$$

and hence,

$$
\sup _{q_{j}(x) \leq 1} q_{j}\left(T^{n} x\right) \leq 2 n
$$

Letting $n \rightarrow \infty$, it follows for every $j \in \mathbb{N}$ that

$$
\limsup _{n \rightarrow \infty} \sqrt[n]{\sup _{q_{j}(x) \leq 1} q_{j}\left(T^{n} x\right)} \leq \lim _{n \rightarrow \infty} \sqrt[n]{2 n}=1
$$

Now, we fix $\lambda \in \mathbb{C}$ with $|\lambda|>1$ and choose $0<c<1$ so that $c|\lambda|>1$. Consequently, for a fixed $j \in \mathbb{N}$, we have by (5.5) that

$$
\limsup _{n \rightarrow \infty} \sqrt[n]{\sup _{q_{j}(x) \leq 1} q_{j}\left(T^{n} x\right)} \leq 1<c|\lambda|
$$

So, there exists $n_{0} \in \mathbb{N}$ such that for every $x \in X$ and $n \geq n_{0}$ we have

$$
q_{j}\left(T^{n} x\right)<c^{n}|\lambda|^{n} q_{j}(x) .
$$

This implies for every $x \in \mathbb{N}$ and $n \geq n_{0}$ that

$$
q_{j}\left(\sum_{m=n}^{\infty} \frac{T^{m} x}{\lambda^{m}}\right) \leq\left(\sum_{m=n}^{\infty} c^{m}\right) q_{j}(x)
$$

where $\sum_{m=n}^{\infty} c^{m} \rightarrow 0$ for $n \rightarrow \infty$ as $0<c<1$. Accordingly, as $j \in \mathbb{N}$ is arbitrary, the series $\sum_{n=0}^{\infty} \frac{T^{n}}{\lambda^{n}}$ is convergent in $\mathcal{L}_{S}(E)$ and so, the operator $R_{\lambda}:=\frac{1}{\lambda} \sum_{n=0}^{\infty} \frac{T^{n}}{\lambda^{n}} \in$ $\mathcal{L}(E)$. In particular, $R_{\lambda}(\lambda I-T)=(\lambda I-T) R_{\lambda}=I$. Thus, $\lambda \in \rho(T)$ and $R(\lambda, T)=$ $R_{\lambda}$. 
Corollary 5.2 Let $E$ be a Fréchet space and $T \in \mathcal{L}(E)$. If $T$ is power bounded, then $\sigma(T) \subseteq \overline{\mathbb{D}}$.

Proof The assumption on $T$ clearly implies that there exists $\lim _{n \rightarrow \infty} \frac{T^{n}}{n}=0$ in $\mathcal{L}_{S}(E)$. So, the result follows from Proposition 5.1.

Remark 5.3 We point out that Proposition 5.1 is a consequence of [10, Proposition 4.4]. Since the proof given here does not depend on the open mapping theorem, the result can be generalized to other contexts. Indeed, arguing as in the proof of Proposition 5.1 we can show that:

Let $E$ be a sequentially complete barrelled lcHs and $T \in \mathcal{L}(E)$. If there exists $\lim _{n \rightarrow \infty} \frac{T^{n}}{n}=$ in $\mathcal{L}_{S}(E)$, then $\sigma(T) \subseteq \overline{\mathbb{D}}$.

The next aim is to extend in the setting of separable Fréchet spaces a result of Jamison [17] about the size of $\sigma_{p}(T) \cap \mathbb{T}$ with $T$ a power bounded operator. In order to do this, we observe that if $T$ is a power bounded operator acting on a Fréchet space $E$, then there exists an increasing sequence $\left\{q_{j}\right\}_{j \in \mathbb{N}}$ of continuous seminorms generating the lc-topology of $E$ such that for every $j, n \in \mathbb{N}$ and $x \in E$ we have

$$
q_{j}\left(T^{n} x\right) \leq q_{j}(x)
$$

Indeed, if $\left\{p_{j}\right\}_{j \in \mathbb{N}}$ is an increasing sequence of continuous seminorms generating the lc-topology of $E$, then for each $j \in \mathbb{N}$ there exist $j^{\prime} \in \mathbb{N}$ with $j^{\prime} \geq j$ and $c_{j}>0$ such that

$$
p_{j}\left(T^{n} x\right) \leq c_{j} p_{j^{\prime}}(x)
$$

for every $x \in E$ and $n \in \mathbb{N}$. If we set

$$
q_{j}(x):=\sup _{n \in \mathbb{N}_{0}} p_{j}\left(T^{n} x\right)
$$

for every $j \in \mathbb{N}$ and $x \in E$, then $\left\{q_{j}\right\}_{j \in \mathbb{N}}$ is an increasing sequence of continuous seminorms generating the lc-topology of $E$ for which (5.6) is satisfied.

Lemma 5.4 Let $E$ be a Fréchet space and $T \in \mathcal{L}(E)$ be a power bounded operator satisfying (5.6). Let $\lambda_{1}, \lambda_{2} \in \sigma_{p}(T) \cap \mathbb{T}$ be independent. Then, if $x_{1}$ and $x_{2}$ are eigenvectors for $\lambda_{1}$ and $\lambda_{2}$ respectively, with $q_{j}\left(x_{1}\right)=q_{j}\left(x_{2}\right)=1$ for some $j \in \mathbb{N}$, we have $q_{j}\left(x_{1}-x_{2}\right) \geq 1$.

Proof We consider the quotient space $X / \operatorname{ker} q_{j}$ and endow it with the canonical quotient norm $\hat{q}_{j}$ defined by

$$
\hat{q}_{j}\left(Q_{j} x\right):=\inf \left\{q_{j}(y): x-y \in \operatorname{Ker} q_{j}\right\}, \hat{q}_{j}\left(Q_{j} x\right)=q_{j}(x),
$$

where $Q_{j}: E \rightarrow \frac{E}{\operatorname{Ker} q_{j}}$ denotes the canonical quotient map. Then $\left(\frac{E}{\operatorname{Ker} q_{j}}, \hat{q}_{j}\right)$ is a normed space. The operator $T$ induces a continuous linear operator $\hat{T}_{j}$ acting on $\frac{E}{\operatorname{Ker} q_{j}}$ 
via (5.6) such that

$$
Q_{j} \circ T=T_{j} \circ Q_{j} \text { and } \hat{q}_{j}\left(T_{j} \hat{x}\right) \leq \hat{q}_{j}(\hat{x}) \text { for all } \hat{x} \in \frac{E}{\operatorname{Ker} q_{j}} \text {. }
$$

Therefore, $T_{j}$ can be continuously extended on the completion $E_{j}$ of $\left(\frac{E}{\operatorname{Ker} q_{j}}, \hat{q}_{j}\right)$, denoted again by $T_{j}$, for which $Q_{j} \circ T=T_{j} \circ Q_{j}$ continues to hold.

We now observe that

$$
T_{j}\left(Q_{j} x_{i}\right)=Q_{j}\left(T x_{i}\right)=Q_{j}\left(\lambda_{i} x_{i}\right)=\lambda_{i} Q_{j}\left(x_{i}\right), \quad i=1,2,
$$

and that, setting $\hat{x}_{i}=Q_{j}\left(x_{i}\right)$ for $i=1,2$, we have

$$
\hat{q_{j}}\left(\hat{x}_{i}\right)=1, \quad i=1,2 .
$$

By [17, Lemma 2] we may conclude $q_{j}\left(x_{1}-x_{2}\right)=\hat{q_{j}}\left(\hat{x}_{1}-\hat{x}_{2}\right) \geq 1$.

Proposition 5.5 Let $E$ be a separable Fréchet space and $T \in \mathcal{L}(E)$ be a power bounded operator. Then $\sigma_{p}(T) \cap \mathbb{T}$ is at most countable.

Proof Let $\left\{q_{j}\right\}_{j \in \mathbb{N}}$ be a fundamental system of seminorms satisfying (5.6).

We set $\Gamma:=\sigma_{p}(T) \cap \mathbb{T}$ and suppose that $\Gamma$ is uncountable. By [17, Lemma 1] $\Gamma$ contains an uncountable set $\Lambda$ such that any two distinct elements of $\Lambda$ are independent. For each $\lambda \in \Lambda$ we select an eigenvector $x_{\lambda}$ corresponding to the eigenvalue $\lambda$ and we set $D:=\left\{x_{\lambda}: \lambda \in \Lambda\right\}$. Of course, $D$ is uncountable. If for each $j \in \mathbb{N}$ we define

$$
D_{j}:=\left\{x \in D: q_{j}(x) \neq 0\right\}
$$

then $D=\cup_{j \in \mathbb{N}} D_{j}$. Moreover, we may suppose without loss of generality for every $j \in \mathbb{N}$ that each element of $D_{j}$ satisfies $q_{j}(x)=1$.

Since $D$ is uncountable, there exists $j_{0} \in \mathbb{N}$ such that $D_{j_{0}}$ is uncountable. We now set $U_{j_{0}}:=\left\{x \in E: q_{j_{0}}(x)<1\right\}$ and $U_{x, j_{0}}(r):=x+r U_{j_{0}}$ for $x \in E$ and $r>0$. Then any two distint elements of $\left\{U_{x, j_{0}}\left(\frac{1}{2}\right): x \in D_{j_{0}}\right\}$ are disjoint sets by Lemma 5.4. This clearly contradicts the separability of $E$. Thus $\Gamma$ is at most countable.

\section{References}

1. Albanese, A.A., Bonet, J., Ricker, W.J.: Mean ergodic operators in Fréchet spaces. Ann. Acad. Sci. Fenn. Math. 34, 401-436 (2009)

2. Albanese, A.A., Bonet, J., Ricker, W.J.: $C_{0}$-semigroups and mean ergodic operators in a class of Fréchet spaces. J. Math. Anal. Appl. 365, 142-157 (2010)

3. Albanese, A.A., Bonet, J., Ricker, W.J.: Gröthendieck spaces with the Dunford-Pettis property. Positivity 14, 145-164 (2010)

4. Albanese, A.A., Bonet, J., Ricker, W.J.: Convergence of arithmetic means of operators in Fréchet spaces. J. Math. Anal. Appl. 401, 160-173 (2013)

5. Albanese, A.A., Bonet, J., Ricker, W.J.: Montel resolvents and uniformly mean ergodic semigroups of linear operators. Quaest. Math. 36, 253-290 (2013) 
6. Albanese, A.A., Bonet, J., Ricker, W.J.: Uniform convergence and spectra of operators in a class of Fréchet spaces. Abstr. Appl. Anal. 2014:179027

7. Albanese, A.A., Bonet, J., Ricker, W.J.: On the continuous Cesàro operator in certain function spaces. Positivity 19, 659-679 (2015)

8. Bonet, J., Frerick, L., Jordá, E.: The division problem for tempered distributions of one variable. J. Funct. Anal. 262, 2349-2358 (2012)

9. Chevalley, C.: Theory of Distributions: Lectures. Columbia University, New York (1951)

10. Fernández, C., Galbis, A., Jordá, E.: Dynamics and spectra of composition operators on the Schwartz space. J. Funct. Anal. 274, 3503-3530 (2018)

11. Fernández, C., Galbis, A., Jordá, E.: Spectrum of composition operators on $\mathcal{S}(\mathbb{R})$ with polynomial symbols. Adv. Math. 365, (2020)

12. Galbis, A., Jordá, E.: Composition operators on the Schwartz space. Rev. Mat. Iberoam. 34, 397-412 (2018)

13. Goliński, M., Przestacki, A.: Dynamical properties of weighted translation operators on the Schwartz space $\mathcal{S}(\mathbb{R})$. Rev. Mat. Compl. 33, 103-124 (2020)

14. Gröthendieck, A.: Produits tensoriels topologiques et espaces nucléaire. Mem. Am. Math. Soc. 16,(1955)

15. Hille, E.: Remarks on ergodic theorems. Trans. Am. Math. Soc. 57, 246-269 (1945)

16. Horvath, J.: Topological Vector Spaces and Distributions, vol. 1. Addison-Wesley Publishing Company, Boston (1966)

17. Jamison, B.: Eigenvalues of modulus 1. Proc. Am. Math. Soc. 16, 375-377 (1965)

18. Köthe, G.: Topological Vector Spaces II. Springer, Berlin (1979)

19. Krengel, U.: Ergodic Theorems, De Gruyter Studies in Mathematics, vol. 6. Walter de Gruyter Co., Berlin (1985)

20. Larcher, J.: Some remarks concerning the spaces of multipliers and convolutors, $\mathcal{O}_{M}$ and $\mathcal{O}_{C}^{\prime}$ of Laurent Schwartz. Rev. R. Acad. Cienc. Exactas Fis. Nat. Ser. A Mat. RACSAM 106, 407-417 (2012)

21. Larcher, J., Wengenroth, J.: A new proof for the bornologicity of the space of slowly increasing functions. Bull. Belg. Math. Soc. Simon Stevin 21, 887-894 (2014)

22. Piszczek, K.: Barrelled spaces and mean ergodicity. Rev. R. Acad. Cienc. Exactas Fis. Nat. Ser. A Math. RACSAM 104, 5-11 (2010)

23. Schwartz, L.: Théorie des distributions, Publications de l'Institut de Mathématique de l'Université de Strasbourg, No. IX-X. Nouvelle édition, entièrement corrigée, refondue et augmentée. Hermann, Paris (1966)

24. Vasilescu, F.-H.: Analytic Functional Calculus and Spectral Decompositions. Editura Academiei. Bucureti and D. Reidel Publ. Comp, Dordrecht (1982)

25. Waelbroeck, L.: Le calcul symbolique dans les algèbres commutatives. J. Math. Pures et Appl. 33, 147-186 (1954)

26. Yosida, K.: Functional Analysis, 6th edn. Springer, Berlin (1980)

Publisher's Note Springer Nature remains neutral with regard to jurisdictional claims in published maps and institutional affiliations. 